\title{
Meinungsumfragen im Journalismus: Nachrichtenwert, Präzision und Publikum
}

\author{
Sibylle Hardmeier
}

Der vorliegende Artikel befasst sich mit der Qualität von Zeitungsberichten im Zusammenhang mit Befragungsresultaten. Mit Hinweis auf die drei Begriffe Nachrichtenwert, Präzision und Publikum werden die unterschiedlichen Anforderungen beleuchtet, mit denen Medienschaffende bei der Redaktion von Befragungsberichten konfrontiert sind. Es wird quantitativ untermauert, dass Meinungsumfragen Nachrichtenwert haben und - auch außerbalb von Wablen - in die mediale Berichterstattung einfließen. Gleichzeitig wird aufgezeigt, dass die Präzision dieser Berichte häufig zu wünschen übrig lässt und zum Teil Nachrichtenwerte durch Febl-und Überinterpretationen konstruiert werden. Deshalb werden anschließend einige Anregungen zur Optimierung der Berichterstattung formuliert. Der Fokus der Darstellung ist auf die Journalistinnen und Journalisten gerichtet. In Umkehrung der Perspektive lassen sich implizit auch Schlussfolgerungen für die Produzentinnen und Produzenten von Befragungsdaten ablesen. In der Annabme jedoch, dass der demoskopische Markt funktioniert, kann man von der Hoffnung ausgeben, dass eine professionelle Nachfrage seitens des Journalismus auch das Angebot der Demoskopie verändert und möglicherweise sogar verbessert.

\section{Meinungsumfragen als News}

Die Verwendung von Resultaten aus der Meinungsforschung gehört heute zur normalen journalistischen Praxis. Massenmedien - und insbesondere Zeitungen - berichten nicht nur in ihrer Rolle als Kommunikateure über Befragungsresultate, welche ihnen die politischen Sprecher (Neidhardt 1994) zukommen ließen, sondern geben auch selbst Meinungsumfragen in Auftrag. Meinungsumfragen stoßen auch in Schweizer Medien auf rege Nachfrage. Diesen Umstand belegen nicht nur die Geschäftszahlen des Verbandes Schweizer Marketing- und Sozialforscher (2000: 8), ${ }^{1}$ sondern auch Analysen der Medienprodukte und Befragungen von Journalistinnen und Journalisten: So hat eine erstmals für die Schweiz durchgeführte Analyse von Tages-, Wochen- und Sonntagszeitungen im zweiten Halbjahr 1997 gezeigt, dass kaum ein Tag vergeht, an dem nicht über Befragungen berichtet wird - und zwar auch dann, wenn man lediglich Berichte erfasst, welche Befragungen zum Hauptaufhänger haben, ${ }^{2}$ wenn man „nur“ politische Themen berücksichtigt ${ }^{3}$ und wenn Phasen der Routinepolitik außerhalb großer natio-

11999 bescherten Verlage, Fernsehen, Radio und Telecom den Schweizer Meinungsforschungsinstituten rund $15 \%$ ihres Umsatzes; wobei zu beachten gilt, dass sich dabei Markt- bzw. Leserforschung und Meinungsforschung der Medien häufig vermischen, weil die Aufträge gekoppelt werden.

2 Es wurden nur solche Berichte aufgenommen, welche in Titel, Lead oder Untertitel direkt oder indirekt auf ein Befragungsresultat aus dem politischen Bereich Bezug nehmen.

3 Die Selektion der, politischen' Berichte erfolgte in zweierlei Hinsicht: Erstens wurde nach einem Pretest entschieden, die Durchsicht der Zeitungen auf Rubriken mit möglichen politischen Inhalten - das heißt die Bereiche wie ,Inland', ,Region und Kanton', ,Ausland' sowie ,Wirt$\mathrm{schaft}^{`}$ - zu begrenzen. Zweitens wurden reine Marktforschungserhebungen ausgeschieden, welche in keinem Bezug zu politischen Entscheidprozessen standen. Zu diesem Zweck wurde 
naler Wahlkampagnen beleuchtet werden. ${ }^{4}$ Zudem wird bestätigt, dass auch die Schweizer Printmedien gerne in die Rolle von Sprechern schlüpfen und über ihre eigenen Umfrageresultate berichten. Weit über ein Drittel (41 Prozent) der Berichterstattung zu Befragungen in diesem Halbjahr befasst sich mit so genannten Media-Polls-Umfragen, die von den Medien selbst in Auftrag gegeben wurden (Hardmeier / Luginbühl 2000).

Für diese Beliebtheit von Befragungsberichten bietet die Wissenschaft verschiedene Erklärungen an. Sie lassen sich unter den drei Begriffen Präzision, Publikum und Nachrichtenwert subsumieren:

- Präzision: Spezialistinnen und Spezialisten journalistischer Berufsrollen verweisen darauf, dass das Instrument der empirischen Sozialwissenschaft helfen kann, das Objektivitätsaxiom im Journalismus zu erfüllen. Recherchen mit und Berichte über Befragungen können demnach dem von Philip Meyer propagierten Ideal des „New Precision Journalism“ (Meyer 1991) nachkommen und sollten ein Berichterstattungsmuster mit hohem Validitätsanspruch widerspiegeln (Weischenberg 1995).

- Publikum: Medienökonomen und Öffentlichkeitssoziologinnen heben zur Erklärung der Beliebtheit des Instruments die Interessen des Publikums hervor. Dabei weist die erste Gruppe auf die Bedürfnisbefriedigung einer Käuferschaft hin; die zweite Gruppe auf die in einer modernisierten, anonymisierten Gesellschaft zentrale Orientierung am „generalisierten Anderen“ (Berger / Berger 1983: 48). Vor diesem Hintergrund bieten publizierte Meinungsumfragen dem Publikum die Möglichkeit, sich über die Meinung anderer Menschen bzw. wichtiger Referenzgruppen einen Überblick zu verschaffen oder die eigenen Ansichten mit diesen zu vergleichen (Mutz 1998).

- Nachrichtenwert: Aus einer konstruktivistischen Perspektive lässt sich argumentieren, dass mit Befragungen vor allem mediale Ereignisse und Schlagzeilen kreiert werden. Weil Befragungen zahlreiche Nachrichtenfaktoren erfüllen können, zum Unterhaltungswert oder zur Erzeugung von Spannung beitragen und unabhängig von äußeren Ereignissen von den Medienschaffenden selbst einsetzbar sind, sind sie bei den Medien so beliebt (Hardmeier / Luginbühl 2000; Stovall / Solomon 1984; Paletz et al. 1980).

Im vorliegenden Bericht wird vor allem auf die erste und zweite Begründung Bezug genommen. Es wird untersucht, inwiefern die Verwendung von Befragungsberichten mit diesen Erklärungsansätzen in Einklang steht und welche praktischen Schlussfolgerungen daraus zu ziehen sind. Dabei wird allerdings die konstruktivistische Argumentation nicht ausgeblendet, und es wird abschließend argumentiert, dass Präzision und Nachrichtenwert auch gleichzeitig einlösbar sind, dass präzise Schlagzeilen zu Befragungsberichten möglich sind. Die Analyse stützt sich auf verschiedene Daten, die im Rahmen des Schwerpunktprogrammes „Zukunft Schweiz" sowie kleineren Forschungsarbeiten am Institut für Politikwissenschaft der Universität Zürich erhoben wurden. Ausgangslage und Basis der Untersuchung bildet die bereits erwähnte Inhalts-

der politische Bezug der Befragung entlang der Begriffsdimensionen ,politics`, ,polity` und ,policies' erfasst; darüber hinaus wurde eine Vercodung für die Ansiedlung der Befragung im politischen Entscheidprozess vorgenommen. Für Fälle, wo das Hintergrundwissen dazu nicht ausreichte, wurde eine Desk-Research gestartet.

4 In der untersuchten Periode fanden keine nationalen Wahlen statt. Im Kontext der vereinzelten lokalen und kantonalen Wahlen sowie des einzigen nationalen Abstimmungstermins vom 28. September 1997 wurden kaum Befragungen publiziert. 
analyse in der zweiten Hälfte 1997 von 29 deutsch-, französisch- und italienischsprachigen Tages-, Wochen- und Sonntagszeitungen in der Schweiz. ${ }^{5}$ Diese inhaltsanalytisch erhobenen Angaben wurden mit Befragungen und Interviews bei den Medienschaffenden ergänzt: zum einen durch 16 qualitative Interviews mit Journalisten und Journalistinnen, welche am Diffusionsprozess von Befragungen beteiligt waren, die sich in unserer Inhaltsanalyse einer besonders großen Beliebtheit erfreuten; ${ }^{6}$ zum anderen mit $\mathrm{Zu}$ satzfragen zur Befragung von Schweizer Medienschaffenden "Schweizer Journalismus unterwegs", welche im Sommer 1998 vom Institut für Medienwissenschaft der Universität Bern und vom Institut für Publizistikwissenschaft und Medienforschung der Universität Zürich ebenfalls im Rahmen des Schwerpunktprogrammes „Zukunft Schweiz“ realisiert worden war.' Obwohl sich der Artikel auf in der Schweiz erhobene Daten stützt, wird davon ausgegangen, dass viele der hier präsentierten Befunde und Schlussfolgerungen verallgemeinerungsfähig und beispielsweise auch auf Deutschland übertragbar sind.

5 Diese Inhaltsanalyse bildet Teil des Projektes „Politische Meinungsumfragen in der politischen Kommunikation: eine Qualitäts- und Wirkungsanalyse“ im Schwerpunktprogramm „Zukunft Schweiz“. Projektleitung: Prof. Dr. S. Hardmeier. Barbara Good hat als Mitarbeiterin dieses Projekts viele der Daten ausgewählt, kodiert und auch verarbeitet. Ihr gilt ein besonderer Dank. Zu Durchführung und Analyse vgl. auch Hardmeier (1999). Die hier präsentierten Daten unterscheiden sich insofern, als ein bereinigter Datensatz von insgesamt 672 Zeitungsberichten verwendet wird.

6 Es handelt sich dabei um Befragungen, die von Medien selbst in Auftrag gegeben wurden. Interviews wurden mit Journalistinnen und Journalisten aus folgenden Zeitungen durchgeführt: Tribune de Genève; Sonntagsblick; Neue Luzerner Zeitung; Berner Zeitung; Le Matin; Coop Zeitung; Sonntagszeitung; Schweizerische Depeschenagentur; Facts; Blick; Tages-Anzeiger Media AG; L'Hebdo; Neue Zürcher Zeitung; Aargauer Zeitung. Die Interviews liegen in transkribierter Form in zwei Forschungsseminararbeiten vor. Vgl. Dufey et al. (1999) sowie Flückiger et al. (1999).

7 Die Studie ging der Frage nach, inwiefern ökonomische, organisatorische und technologische Imperative Auswirkungen auf die journalistische Produktion in den Schweizer Medien haben. Die Befragung basierte auf einem schriftlichen Fragebogen. Das Sampling Frame bildete die Mitgliederliste der großen Berufsverbände SVJ, SJU und SSM (insgesamt 9135 Mitglieder). Daraus wurde eine geschichtete Zufallsauswahl von 5404 Personen gezogen. Der Rücklauf auswertbarer Fragebogen belief sich auf 2111; nach Bereinigung ergab sich die endgültige Fallzahl von insgesamt 2020 Medienschaffenden. Darin waren Befragte aus dem Printbereich zu $66 \%$ vertreten, Befragte vom öffentlichen und privaten TV zu 28 \%. Vgl. Marr / Wyss (1999). Da im Rahmen dieser Erhebung auch Medienschaffende befragt wurden, die mit großer Wahrscheinlichkeit kaum je in die Vergabe bzw. journalistische Umsetzung von politischen Meinungsumfragen involviert sind (z. B. Fotografen oder Filmkritikerinnen), wurde für den Vergleich mit unserer Inhaltsanalyse mit einem reduzierten Datensatz gearbeitet. Dieser Datensatz umfasst 428 Fälle und enthält Daten von Medienschaffenden, die a) bei Medien beschäftigt sind, die den in der Medieninhaltsanalyse untersuchten entsprechen; die b) in Ressorts arbeiten, die im Rahmen der Medieninhaltsanalyse ebenfalls erhoben wurden; und die c) eine schreibende Funktion innehaben. Bei einer Irrtumswahrscheinlichkeit von $5 \%$ und einer Antwortverteilung von 50 \%: 50 \% beträgt der Stichprobenfehler für dieses Sample \pm 4,7 Prozentpunkte. 


\section{Präzision oder Publikum? Wie und weshalb Journalisten und Journalistinnen über Meinungsumfragen berichten}

\subsection{Der Validitätsanspruch der Medienschaffenden}

Der von Philip Meyer zu Beginn der 70er Jahre erstmals propagierte „Präzisionsjournalismus" versteht den journalistischen Beruf als Wissenschaft. Er verlangt, dass im Journalismus wissenschaftliche Methoden angewendet werden und reklamiert wissenschaftliche Objektivitätskriterien für den gesamten Prozess der massenmedialen Kommunikation (Meyer 1991: 6). Entsprechend wird in der von Weischenberg entwickelten Typologie das journalistische Berichterstattungsmuster des Präzisionsjournalismus durch ein Rollenbild als Forscher, mit hohen Kompetenz- und Validitätsansprüchen sowie einer wissenschaftlichen Berufsethik charakterisiert (Weischenberg 1995: 114). Theoretisch lässt sich folglich annehmen, dass das sozialwissenschaftliche Instrument „Befragung“ zum typischen Instrument eines Präzisionsjournalisten zählt und Journalistinnen, welche sich auf demoskopische Daten ${ }^{8}$ abstützen, einen hohen Validitätsanspruch an den Tag legen.

In unserer Analyse haben wir dieses Berichterstattungsmuster und Rollenverständnis inhaltsanalytisch erfasst: In Anlehnung an vergleichbare Studien aus den USA, Deutschland, Holland oder Israel (Brettschneider 1997; de Boer 1995; Brady and Orren 1992; Miller et al. 1991; Rollberg et al. 1990; Weimann 1990: 401; Salwen 1985; Miller / Hurd 1982) wurde zum einen untersucht, inwiefern als zentrales Kriterium der Objektivität „Intersubjektivität von journalistischen Realitätsbeschreibungen“ (Donsbach 1991: 27) in den Befragungsberichten gewährleistet ist und die befragungstechnisch relevanten Angaben vermittelt werden. Zum anderen wurde aber erstmals und in Ergänzung zu dieser formalen Qualitätsanalyse betrachtet, ob mit Befragungsdaten auch entsprechend kompetent umgegangen wird. Da es sich hierbei um demoskopische Daten handelt, die in einem Stichprobenverfahren und mittels standardisierter Interviews erhoben wurden, ${ }^{9}$ wurde bei der Identifizierung möglicher Schwachstellen auf die zentralen Bestimmungsgrößen einer Stichprobe rekurriert: das Vertrauensintervall einer Stichprobe, ${ }^{10}$ die

8 Zur Definition von Demoskopie vgl. Kaase (1994: 73): Unter Demoskopie wird „die empirische Untersuchung von repräsentativen Querschnitten (Sample) der [...] Bevölkerung mittels standardisierter Erhebungsinstrumente und mündlicher Befragungen" verstanden.

9 In der Medieninhaltsanalyse wurden nur Artikel erfasst, welche sich auf solche Befragungsdaten stützten. Artikel bzw. Daten, die mit anderen Erhebungsinstrumenten wie Straßenumfragen, Expertenbefragungen o. Ä. erfasst wurden, wurden nicht berücksichtigt.

10 Jede noch so gute Stichprobe bleibt mit einem Unsicherheitsfaktor behaftet. Es stellt sicher daher immer die Frage, inwiefern die in einer Stichprobe ermittelten Werte den tatsächlichen oder wahren Werten in der Grundgesamtheit entsprechen. Das Vertrauensintervall oder der Stichprobenfehler ist ein Kriterium dafür. Damit wird der Bereich angegeben, innerhalb dessen der wahre Wert liegt, und die vereinfachte Formel $\left( \pm 1.96 \sqrt{ } \mathrm{p}^{*} \mathrm{q} / \mathrm{n}\right)$ zeigt, dass sich der Wert aufgrund der von den Forscherinnen und Forschern festgelegten Irrtumswabrscheinlichkeit, der Antwortverteilung (p, q) sowie der Stichprobengröße (n) berechnen lässt. Für die Irrtumswahrscheinlichkeit wird meistens der Grenzwert von $5 \%$ gewählt, sodass man mit 95-prozentiger Wahrscheinlichkeit eine korrekte Aussage machen kann (der Wert 1,96 steht dafür). Entsprechend würde beispielsweise bei einer Stichprobe von 1000 Befragten und einem Wert von $50 \%$ und einem Vertrauensintervall von \pm 3.1 Prozentpunkten die korrekte Angabe zum Vertrauensintervall lauten: „Mit einer Wahrscheinlichkeit von 95\% liegt der tatsächliche Anteil im Be- 
Definition von Grundgesamtheit und Stichprobe ${ }^{11}$, die Antwortverteilungen im Sinne von relativen und absoluten Mehrheitsverhältnissen ${ }^{12}$ sowie die Signifikanz eines $\mathrm{Zu}$ sammenhangs bzw. Unterschieds oder einer Veränderung. ${ }^{13}$ Diese vier statistischen Faktoren gelten als wichtige Indikatoren für die Genauigkeit einer Erhebung und sind deshalb auch für die journalistische Interpretation und Aufbereitung der Befragungen zentral. ${ }^{14}$

reich von 46,9 und 53,1 Prozent“. Zudem ergibt sich aus dieser Formel: je deutlicher die Antwortverteilung (z. B. $90 \%: 10 \%$ ), desto einfacher ist es, präzise Angaben zu machen (weil das Vertrauensintervall kleiner wird). Wenn hingegen bei den Antworten eine Pattsituation von $50 \%: 50 \%$ eintritt, die Ansichten unter den Befragten also sehr polarisiert sind, ist es am schwierigsten zu sagen, wie der wahre Wert in der Grundgesamtheit aussieht. Eingebürgert hat sich deshalb, vom schlechtesten Fall (50\%: 50 \%) auszugehen. Wenn in einem Stichprobenbeschrieb gesagt wird, der „Stichprobenfehler beträgt maximal \pm 3.1 Prozentpunkte“, dann bezieht sich diese Aussage auf eine Antwortverteilung von $50 \%: 50 \%$. Wenn die Antwortverteilung viel ungleicher, zum Beispiel 10\%: 90\% ausfällt, dann würde das Vertrauensintervall nur noch $\pm 1,9$ Prozentpunkte betragen (wiederum bei einer Stichprobengröße von 1000 Befragten).

11 Eine Stichprobe zeichnet sich dadurch aus, dass keine Vollerhebung realisiert wird. In der Stichprobentheorie wird deshalb zwischen drei verschiedenen Erhebungseinheiten unterschieden. Unter der (angestrebten) Grundgesamtheit oder Population werden jene Personen oder Einheiten verstanden, auf die sich die Aussagen der Erhebung beziehen sollen. Aus praktischen Gründen ist diese Grundgesamtheit häufig nicht ganz identisch mit der Erbebungsgrundgesamtheit oder Auswahlgesamtheit aus welcher faktisch die eigentliche Stichprobe gezogen wird. Zentral ist, dass mit der Stichprobe nur über jene Einheiten etwas ausgesagt werden kann, welche auch in der Grund- oder Auswahlgesamtheit vertreten sind. Will man beispielsweise etwas über die Schweizer Bevölkerung aussagen, befragt indessen lediglich 18- bis 55-jährige Stimmberechtigte, so sind gewisse Altersgruppen sowie die ausländische Bevölkerung nicht vertreten. In diesem Falle handelt es sich um ein „undercoverage“, gewisse Einheiten, die zwar in der Grundgesamtheit, nicht aber in der Auswahlgesamtheit sind, können nicht in die Stichprobe gelangen. Enthält umgekehrt die Stichprobe Einheiten, die nicht zur Grundgesamtheit oder Zielgruppe der Erhebung gehören, spricht man von „overcoverage“.

12 Wie bereits in Fußnote 10 erläutert, sind die Antwortverteilungen bei einer Stichprobe eine zentrale kritische Größe. Im vorliegenden Fall wurde ein einfaches Gütekriterium für journalistisch vermittelte Antwortverteilungen und Mehrheitsverhältnisse gewählt. Die Frage nämlich, ob eine gewisse Antwortkategorie absolut am meisten Nennungen enthält oder vielleicht nur im Vergleich zu den anderen Antwortkategorien.

13 Die schließende Statistik richtet ihre Aufmerksamkeit nicht nur auf die Fehlerbereiche einer Stichprobe (Vertrauensintervall), sondern auch auf die Prüfung von Zusammenhängen zwischen Variablen. Mit Signifikanztests können solche Zusammenhänge getestet werden. Konkret geht es dabei um die Frage, ob die Abweichungen, die man in einer empirischen Tabelle feststellt, zufällig oder nicht mehr zufällig, also statistisch signifikant sind. Getestet wird die Nullhypothese, die besagt, dass zwischen zwei Variablen kein Zusammenhang besteht. Die Signifikanz ist allerdings mit bloßem Auge nicht immer erkennbar; je nach Antwortverteilung und Größe der Stichprobe sind bereits kleine Differenzen signifikant oder große Differenzen kommen noch per Zufall zustande. Der Terminus, fragliche Signifikanz' verweist darauf, dass keine eigentlichen Berechnungen vorgenommen werden konnten, da der Artikel selbst die Kodiereinheit bildete. Die Signifikanz wurde bei geringen Unterschieden zwischen Subgruppen oder in der Zeit und insbesondere bei kleinen Fallzahlen in Frage gestellt.

14 Mit diesen vier Kriterien wird bewusst auf eine der möglichen Fehlerquellen bei Befragungen fokussiert: den Samplingfehler. Die andere Fehlerquelle von Befragungen auf der Ebene der eigentlichen Messung bleibt ausgeklammert, da sie in einer quantitativen Inhaltsanalyse kaum zu erfassen ist. Zu den beiden Fehlerquellen von Befragungen vgl. das Standardwerk von Groves (1989). 
Tabelle 1: Angabe von methodischen Informationen in Artikeln mit unterschiedlichem Status und Präsentationsstil (Medieninhaltsanalyse)

\begin{tabular}{|c|c|c|c|}
\hline Berufsethische Norm & $\begin{array}{l}\text { Hauptartikel } \\
\qquad(\mathrm{n}=399) \\
\%\end{array}$ & $\begin{array}{l}\text { Sekundärberichte } \\
\qquad(\mathrm{n}=201) \\
\%\end{array}$ & $\begin{array}{c}\text { Hauptartikel } \\
\text { mit Infobox } \\
(\mathrm{n}=74) \\
\%\end{array}$ \\
\hline Durchführendes Institut $^{a}$ & 81 & $50 \% *$ & $95 \%$ \\
\hline Stichprobengröße $\mathrm{e}^{\mathrm{ab}}$ & 51 & $64 * *$ & $91^{++}$ \\
\hline Auftraggeber ${ }^{a}$ & 48 & $82 \div *$ & $81 * *$ \\
\hline Erhebungsdatuma & 42 & $37 * *$ & $59 * \%$ \\
\hline Zusammensetzung der Stichprobe ${ }^{a}$ & 32 & n. c. & $58 * \%$ \\
\hline Frageformulierung ${ }^{\mathrm{ab}}$ & 35 & n. c. & $88^{++}$ \\
\hline Erhebungsmethode ${ }^{\mathrm{ab}}$ & 10 & $7^{\text {n.s. }}$ & $19^{++}$ \\
\hline Basiszahlen für die Prozentberechnunga & 10 & n. c. & $1 *$ \\
\hline Stichprobenziehunga & 4 & $0 \%$ & $8^{++}$ \\
\hline Angaben zur Ausschöpfunga & 0 & n. c. & On.s. \\
\hline Angaben zu Stimmberechtigten im Sample ${ }^{\mathrm{b}}$ & 26 & n. c. & $34^{++}$ \\
\hline Größe der Subgruppen ${ }^{b}$ & $9^{c}$ & n. c. & $38^{++}$ \\
\hline Stichprobenfehler ${ }^{\mathrm{b}}$ & 4 & n. c. & $19^{++}$ \\
\hline Stichprobenfehler für Subgruppen ${ }^{\mathrm{b}}$ & $4^{\mathrm{c}}$ & n. c. & $19^{++}$ \\
\hline [Angaben zu etwaigen Gewichtungen ${ }^{a b}$ & 2 & n. c. & $\left.12^{++}\right]$ \\
\hline
\end{tabular}

Hauptartikel sind definiert als Artikel, in denen Umfrageresultate zum ersten Mal veröffentlicht werden - unabhängig von der Auftraggeberschaft. Sekundärberichte sind definiert als Berichte, die auf bereits publizierte Meinungsumfragen Bezug nehmen. Für den zweiten Typus von Berichten wurden nur ausgewählte Standards kodiert.

Eine Infobox ist ein separates Kästchen mit methodischen Angaben.

Die Prozentwerte sind auf ganze Zahlen gerundet worden; Abweichungen vom Total sind auf Rundungen zurückzuführen.

n. c. nicht codiert (Sekundärberichterstattung).

a Berufsethische Normen, Swiss Interview (1989).

b Richtlinien zur Durchführung von abstimmungs- und wahlbezogenen Umfragen, Swiss Interview (1993). Genau genommen greifen diese Richtlinien nur, wenn die Publikation der Befragungsdaten vor einer Wahl oder Abstimmung erfolgt; das sind im vorliegenden Sample lediglich 5 Hauptartikel. Von diesen machen 80 \% (4) bzw. 40 \% (2) Angaben zu den Stimmberechtigten im Sample bzw. zum Stichprobenfehler. Angaben zu Größe der Subgruppen bzw. dem Stichprobenfehler für diese Subgruppen werden in keinem Fall gemacht. 1

c Diese Prozentangaben basieren auf Artikeln, die über Resultate von Subgruppen berichten.

** jeweilige Differenz zu Hauptartikeln stark signifikant $(\mathrm{p}<0.01)$.

$++\quad$ jeweilige Differenz zu Hauptartikeln stark signifikant $\left(\mathrm{p}<0.01\right.$, exakte Signifikanz). ${ }^{2}$

n. s. nicht signifikant.

Quelle: SPP Projekt Meinungsumfragen (Hardmeier).

$1 \quad$ Vgl. Swiss Interview (1993: 1). Mittlerweile wurden die Richtlinien revidiert, vgl.: Verband Schweizer Marketing- und Sozialforscher (1999).

2 Exakte Tests bzw. das Monte-Carlo-Verfahren werden für kleine, unausgewogene Verteilungen verwendet. 
Die vorliegenden Daten demonstrieren, dass man bei der medialen Aufbereitung von Befragungsberichten sicher nicht von Präzisionsjournalismus sprechen kann. Denn zum einen bestätigt sich das Bild, das sich aus Analysen in anderen Ländern bereits abgezeichnet hat: Die Medienschaffenden stellen nur beschränkt intersubjektive Nachvollziehbarkeit her und kommunizieren die methodischen Angaben über repräsentative Befragungen nur zum Teil (vgl. Tabelle 1). Es werden eher die einfacheren, wenig technischen Aspekte wie das durchführende Institut, die Stichprobengröße, der Auftraggeber oder das Erhebungsdatum angegeben; die komplizierteren Sachverhalte wie die Erhebungsmethode (telefonisch, Face-to-Face, schriftlich), die Stichprobenziehung, der Stichprobenfehler oder die Ausschöpfungsquote werden hingegen meist übergangen (vgl. Tabelle 1, Spalte 1 sowie Hardmeier 1999: 264). Eine zusätzliche Einbuße erleidet die Transparenz zudem in Sekundärberichten.

Gleichzeitig lässt sich nachweisen, dass die Genauigkeit der Erhebungen in den Medienberichten häufig strapaziert und über Mehrheiten berichtet wird, wo dies aufgrund der Erhebungsmethode nicht zulässig ist (vgl. Tabelle 2): In 45 Prozent aller erfassten Artikel sind solche Interpretationsfehler feststellbar. Dabei gilt es zu berücksichtigen, dass Fehlinterpretationen inhaltsanalytisch umso besser zu erfassen sind, je mehr technische Angaben zur jeweiligen Befragung gemacht werden. Vor dem Hintergrund dieser methodischen Restriktion sind deshalb vor allem zwei Schlussfolgerungen zum Umgang der Medien mit Befragungen zulässig: Allein die Wiedergabe methodologischer Angaben schützt nicht automatisch vor Fehl- und Überinterpretationen. Weil zudem Fehlinterpretationen mit mehr Detailinformationen verknüpft sind und prominent aufgemachte Berichte mehr Angaben enthalten, sind Fehlleistungen in Artikeln, in welchen die Printmedien über ihre eigens in Auftrag gegebenen Befragungen berichten, überdurchschnittlich oft erkennbar. Offensichtlich führen hier die Effekte einer transparenteren Berichterstattung einerseits, das Bedürfnis nach einer Schlagzeile für die eingekaufte Befragung andererseits zu einer negativen Kumulation. In 66 Prozent der Berichte über Befragungen, die von den Medien in Auftrag gegeben wurden, sind ein oder mehrere Interpretationsfehler feststellbar. ${ }^{15}$

Aus Tabelle 2 geht hervor, was auch stichprobentheoretisch begründet ist: Die Kumulation der unterschiedlichen Interpretationsfehler ist möglich, häufig auch logisch verknüpft, aber keineswegs zwingend. Die ersten vier Typen von Fehl- oder Überinterpretationen tangieren das, was in der medialen Berichterstattung als (vermeintliche) Mehrheitsverhältnisse oder Gegensätze geschildert wird.

\section{Schlagzeilen mit fraglichen Mebrheitsverbältnissen}

Beispielhaft für Schlagzeilen mit fraglichen Mehrheitsverhältnissen steht ein Bericht der Zeitung „Blick“. Diese titelte im Rahmen einer mehrteiligen „Europa-Erhebung“ im Herbst $1997^{16}$ auf der Frontseite der Ausgabe vom 30. September 1997 in fetten Let-

15 Cramer's V = $0.12(\mathrm{p}<0.05) ; \mathrm{n}=368$ Artikel, in denen die Auftraggeberschaft identifiziert werden konnte. 71 Artikel, bei denen eines der im Sample erfassten Medien als Auftraggeber figurierte.

16 Diese Befragungsserie zeichnete sich durch die Kumulation von Fehlinterpretationen aus: ZweiArtikel weisen gleichzeitig 4 der erfassten Fehlinterpretationen auf (vgl. „Blick“; 30. September bzw. 2. Oktober 1997); ein anderer Blickartikel hält zudem in unserem Sample den negativen Rekord von 5 unterschiedlichen Interpretationsfehlern in einem Text (Blick, 1. Oktober 1997). 
Tabelle 2: Statistisch begründete Feblinterpretationen in der journalistischen Berichterstattung (Medieninhaltsanalyse)

\begin{tabular}{llr}
\hline Typen der Fehlinterpretationen & $\%$ & $\mathrm{n}$ \\
\hline Mehrheitsverhältnisse vs. Vertrauensintervall im Lauftext & $11(4)$ & 73 \\
Mehrheitsverhältnisse vs. Vertrauensintervall in Titel / Lead & $5(1)$ & 31 \\
relative vs. absolute Mehrheiten & 7 & 45 \\
fragliche Signifikanz eines Gegensatzes oder einer Veränderung & $20(7)$ & 134 \\
Stichprobe vs. Grundgesamtheit & 22 & 148 \\
\hline Kumulation von Feblinterpretationen & $\%$ & $n$ \\
\hline 1 von 5 codierten Fehlern & 31 & 207 \\
2 von 5 codierten Fehlern & 11 & 74 \\
3 von 5 codierten Fehlern & 3 & 17 \\
4 von 5 codierten Fehlern & 1 & 5 \\
5 von 5 codierten Fehlern & 0 (gerundet) & 1 \\
Total mit codierten Fehlern & $45^{*}$ & 304 \\
kein(e) Fehler & 55 & 368 \\
\hline
\end{tabular}

Lesebeispiel: Insgesamt weisen 304 von 672 Artikeln einen oder mehrere Interpretationsfehler auf, also 45 Prozent. In 368 Artikeln (55 Prozent) ist keiner der aufgeführten Interpretationsfehler feststellbar. ${ }^{1}$

* Abweichungen vom Total (46\%) ergeben sich aufgrund von Rundungen.

Die Angaben in der 2. Spalte in Klammern geben die Prozentwerte für jene Fälle an, wo aufgrund des Medienberichts oder der Institutsdokumentation eindeutig feststellbar ist, dass es sich um eine reine Zufallsauswahl handelte oder wo ein Vertrauensintervall direkt angegeben wurde. Das heißt, es kann sein, dass in den anderen Fällen (Quota oder Random/Quota) aus stichprobentheoretischer Sicht eigentlich nicht von einem Stichprobenfehler bzw. einer Irrtumswahrscheinlichkeit gesprochen werden darf, weil die Annahmen der Zufallsauswahl nicht erfüllt sind. Die Berechnung des Vertrauensintervalls liefert aber auch bei diesen Fällen einen Hinweis dafür, dass im Bericht eine Punktgenauigkeit suggeriert wird, die mit einer Befragung nicht erreicht werden kann.

Quelle: SPP Projekt Meinungsumfragen (Hardmeier)

1 Die Codierung dieser Interpretationsfehler erfolgte durch eine sozialwissenschaftlich ausgebildete Person. Die Codierungen wurden ex post einem Test für Intercodierer-Reliabilität basierend auf 207 Artikeln unterzogen. Die Übereinstimmung erwies sich bei allen vier Variablen als zufrieden stellend (97.1 - 89.7 Prozent Übereinstimmung). Die Berechnung des einfachen Prozentsatzes scheint uns legitim, weil die Fehlerinterpretationen als Stringvariable aufgenommen wurden, die Codierung also sozialwissenschaftlich begründet werden musste, und die Dichotomisierung in zwei Kategorien erst nachträglich erfolgte. Vgl. Stierli (2000 A$10 \mathrm{f}$.$) .$

tern: „Schweizer wollen in die EU - sofort! 56,4 \% dafür; Ständemehr“. Aus der Berichterstattung geht indessen hervor, dass pro Kanton lediglich 100 Personen $^{17}$ befragt worden waren. Damit kann über die zentrale Frage der Mehrheit der Stände ${ }^{18}$ nichts Verlässliches gesagt werden - schon gar nicht auf die Kommastelle genau. ${ }^{19}$ Wie ein Beispiel

17 Es handelte sich um eine telefonische Befragung. Die Art der Stichprobenziehung wird nicht bekannt gegeben; darüber, ob die Befragten stimmberechtigt sind, sagt der technische Bericht nichts aus. Der Auftraggeber hat uns keine Einsicht in die Untersuchungsmaterialien gewährt.

18 Im Falle eines Staatsvertrags oder eines obligatorischen Referendums muss eine Vorlage nicht nur die Hürde des Volksmehrs, sondern auch des Ständemehrs nehmen.

19 Diese vermeintliche Punktgenauigkeit wurde für alle Hauptartikel erfasst. Sie ist in $21 \%$ der Artikel, welche numerische Wiedergaben verwenden, anzutreffen $(n=362$ Hauptartikel, welche numerische Angaben machen). 
aus der „Tribune de Genève“ zeigt, sind auch andere Zeitungen mit diesem Problem von vermeintlich punktgenauen und eindeutigen Majoritäten konfrontiert. Anlässlich einer Befragung bei 500 stimmberechtigten Genferinnen und Genfern war auf der Frontseite dieser Zeitung zu lesen: „Umfrage: Die Linke erhält Mehrheit im Großen Rat“ ${ }^{20}$ Genau genommen - und auf der Innenseite der Zeitung auch nachzulesen - erhielten aber die linken Parteien bei den Befragten lediglich 52 Prozent der Stimmen. ${ }^{21}$ Wenn man dabei in Betracht zieht, dass die Unentschiedenen bei der Berechnung dieser Prozentwerte zu Recht - unberücksichtigt blieben, dann spitzt sich das Problem der Interpretation über den Stichprobenfehler noch zu: Der Unschärfebereich ist dann nämlich noch größer als in der Zeitung für das Gesamtsample angegeben (4.5 Prozentpunkte). ${ }^{22}$

\section{Fragliche Mebrheiten im Lauftext}

Präsentationen von vermeintlich eindeutigen Mehrheiten können mit Überinterpretationen im Lauftext einhergehen. ${ }^{23}$ Die vergleichsweise hohe Anzahl von solchen Fehlinterpretationen im Lauftext leitet sich aber vor allem aus folgendem Muster ab: Insgesamt sind Titel oder Lead in diesen Berichten nicht ausgeprägt „reißerisch“, an einzelnen Stellen im Lauftext wird indessen die Verwendung des Mehrheitsbegriffs wenig reflektiert. Beispielhaft dafür steht ein Bericht aus dem „Corriere del Ticinio“ nach einer Befragung der Televisione Svizerra Italiana über Europa. Unter dem Titel „Europabefürworter nehmen zu“ ist dort zu lesen: „Die Befürworter eines Europabeitritts stellen [...] nunmehr die Mehrheit. $51 \%$ der Schweizer würden heute im Falle einer Abstimmung mit Ja antworten, $38 \%$ sind dagegen und $11 \%$ noch unentschieden. “24 Angesichts der knappen Mehrheit und des recht hohen Anteils von Unentschiedenen ist das doch eine recht gewagte Behauptung.

\section{Absolute und relative Mebrheiten}

Eng verknüpft mit dem Problem der unsicheren Mehrheitsverhältnisse aufgrund des Stichprobenfehlers können der Umgang mit relativen und absoluten Mehrheiten bei den Antwortverteilungen sowie mit der Signifikanz von Zusammenhängen sein. Dabei wird im ersten Fall häufig einfach unterschlagen, dass es sich bei der berichteten Majorität höchstens um relative Mehrheiten handelt. Dafür ein Beispiel aus der „Neuen Luzerner Zeitung " zur Verkehrssituation in der Stadt Luzern: Ein großes Kuchendiagramm auf der Frontseite der Zeitung gibt die Beurteilungen der Befragten zur Verkehrssituation wieder und enthält folgende Kategorien: „sehr gut“ (2 Prozent), „gut“ (27 Prozent), ,soso“ (31 Prozent), „schlecht“ (27 Prozent), „sehr schlecht“ (12 Prozent) sowie „Unent-

20 Tribune de Genève, 23. September 1997, Original: „Sondage: la gauche obtient la majorité au Grand Conseil“.

21 Die Resultate wurden von der Zeitung auf die Kommastelle genau angegeben; hier also 52,3 \%.

22 Dieses Vertrauensintervall gilt lediglich für das Gesamtsample; bei der Analyse von kleineren Subgruppen wird der Wert bzw. Unschärfebereich größer.

23 Bei 84 Prozent der Artikel, welche das Problem im Titel, Untertitel oder Lead aufweisen, ist die gleiche Fehlinterpretation auch im Lauftext ersichtlich.

24 Corriere del Ticino, 25. November 1997. Darüber hinaus fallen in diesem Artikel auch ein unsauberer Umgang mit der Grundgesamtheit sowie eine Strapazierung der Daten in Bezug auf Signifikanzen und mögliche Veränderungen auf. Der Originaltext lautet: „Eurofavorevoli“ in aumento” (Titel). „I favorevoli all'Europa seconda Gerosa sono ormai la maggioranza. Il $51 \%$ di svizzeri in caso di votazione risponderebbero adesso di si, contro il $38 \%$ di no e il $11 \%$ di indecisi." Gerosa ist ein in der italienischen Schweiz tätiger Demoskop. 
schiedene und Antwortverweigerungen“ (1 Prozent). Obwohl sich daraus lediglich eine eindeutig ungenügende Bewertung ("schlecht“ und „sehr schlecht“) von zusammen genommen 39 Prozent ergibt, lautete der Titel des Berichts „Situation ist ,eher schlecht"“ und in der Legende zur Grafik wurde gefolgert: „Die Demoscope-Umfrage zeigt, dass die Mehrheit mit der Verkehrssituation in der Stadt unzufrieden ist. "25 Dabei ist offensichtlich, dass es sich hier höchstens um eine relative Mehrheit handelt - man hätte also genauso die Legende „Mehrheit findet die Situation mäßig gut“ wählen können. Aus identischen Gründen lässt sich auch der Untertitel zu einem Bericht im Nachrichtenmagazin „Facts“ hinterfragen, wonach eine „Mehrheit der Schweizer Beschäftigten“ bereit sei, „Arbeitszeit und Lohn zu reduzieren“. Die 59 Prozent der befragten VollzeitBeschäftigten, welche angeblich für die Einführung der 37-Stunden-Woche und entsprechende Lohneinbussen sind, stellen nämlich bei genauerem Hinsehen keine besonders homogene Gruppe dar. Liest man doch im Lauftext, „44 Prozent der Befragten befürworten die Arbeitsverkürzung vorbehaltlos, 15 Prozent unter gewissen Bedingungen“. ${ }^{26}$

\section{Fragliche Signifikanz}

Genauso wie es bei einer Gesamtstichprobe einen Unschärfebereich gibt, muss auch bei Aussagen über Differenzen zwischen einzelnen Subgruppen oder über die Zeit hinweg in Betracht gezogen werden, dass diese Unterschiede vielleicht nur in der Stichprobe, jedoch nicht in der Realität existieren. So ist beispielsweise die Signifikanz des in der „Berner Zeitung“ vom 31. Juli 1997 berichteten Gegensatzes zwischen Romandie und Deutschschweiz mehr als fraglich: „Die Westschweizer optimistischer als Deutschschweizer: $51 \%$ der Welschen rechnen in den nächsten 12 Monaten mit einem leichten oder spürbaren Aufschwung der Wirtschaft. Diese Ansicht teilen nur $48 \%$ der Deutschschweizer.“

\section{Grundgesamtheit und Stichprobe}

Da das Ziel einer Stichprobe darin besteht, etwas über die gesamte Zielgruppe auszusagen und von einer beschränkten Zahl von Befragten auf die gesamte Grundgesamtheit zu schließen, ist auch das Verhältnis von Grundgesamtheit und Stichprobe für die Qualität und Interpretation einer Befragung bedeutsam. Nun zeigt sich, dass in der journalistischen Berichterstattung diese Einheiten recht häufig nicht übereinstimmen (vgl. Tabelle 2, 22 Prozent). In diesen Artikeln werden Rückschlüsse auf eine Zielgruppe vorgenommen, die aufgrund der Zusammensetzung der Befragten eigentlich nicht zulässig sind. Besonders typisch scheint dabei der Rückschluss von einer eng definierten Stichprobe auf „die Schweizerinnen und Schweizer“ oder „die Bevölkerung“. Zum Beispiel, wenn von einem Sample stimmberechtigter Schweizerinnen und Schweizer - was im Kontext von Wahlen und Abstimmungen durchaus Sinn machen kann - auf die „Schweizer Bevölkerung“ oder das „Volk“ geschlossen wird. ${ }^{27}$ Oder wenn zum Beispiel

25 Neue Luzerner Zeitung, 17. November 1997.

26 Facts, 23. Oktober 1997. Wenn schließlich 60 Prozent dieser vermeintlichen Mehrheit bekunden, sie wären bereit, für die Stundenreduktion auch eine Lohneinbuße hinzunehmen, so dürften das noch rund 35 Prozent der Gesamtstichprobe von 600 Befragten sein.

27 Neue Zürcher Zeitung, 16. Oktober 1997, mit dem Titel „Getrübte Sicht vom Schuldenberg; Das Volk kennt die Fakten, nicht aber die Zusammenhänge“. 
bei einer Stichprobe von Personen über 18 Jahren aus dem deutsch- und französischsprachigen Landesteil von „den Schweizern“ gesprochen wird. ${ }^{28}$

Insgesamt zeigt die vorliegende Analyse, dass die journalistische Aufbereitung von Befragungsresultaten häufig unpräzise ist und fragwürdige Dateninterpretationen aufweisen kann. Mit der Einhaltung und Befolgung von - wie auch immer gestalteten - Publikationsstandards geht keineswegs zwingend und automatisch eine Berichterstattung einher, welche dem Ideal des „scientific journalism“ entspricht. Entsprechend selten finden wir einen Umgang mit Befragungsdaten, der als kritisch bezeichnet werden kann und einen sozialwissenschaftlichen Validitätsanspruch offen legt, wie er vom Verfasser des „New Precision Journalism“ reklamiert oder in journalistischen Rollenbildern festgehalten wird. ${ }^{29}$

\subsection{Die Publikumsorientierung der Medienschaffenden}

Wenn nicht der sozialwissenschaftliche Validitätsanspruch des Präzisionsjournalismus die mediale Berichterstattung über politische Meinungsumfragen prägt, dann könnte es vor allem auch die Publikumsorientierung der Medienschaffenden sein. Auf diese Möglichkeit wurde vor allem auch in den qualitativen Interviews hingewiesen. So hielt Journalist K. beispielsweise fest: „Die Leute wissen auch gerne, ob sie mit ihrer Meinung über ein Thema im Trend liegen oder nicht“ (vgl. Dufey et al. 1999: A-23). Journalist F. wiederum führte in einem ähnlichen Zusammenhang aus: „Andererseits sehen sich die Leute gerne widerspiegelt in einer Zeitung. Wenn man liest, dass jeder dritte Berner am Sonntag bei einer Tankstelle Zigaretten einkaufen geht, dann erkennt sich ein großer Teil der Leute wieder in der Zeitung" (vgl. Flückiger et al. 1999: 89). Diese Einschätzung wird offensichtlich von weiten Teilen der schreibenden Journalistinnen und Journalisten geteilt (vgl. Tabelle 3): Der Aussage „das Interesse der Bevölkerung an repräsentativen Meinungsumfragen und Befragungsresultaten ist groß" stimmt eine Mehrheit von 65 Prozent zu (Kategorien 4 - 6). Die höchsten Prozentwerte finden sich in den Kategorien 4 und 5, die Kategorie 6 („volle Zustimmung“) vereinigt noch 9 Prozent der Antworten auf sich.

Über die Hälfte der antwortenden Journalistinnen und Journalisten geht also davon aus, dass bei der Bevölkerung vielleicht kein brennendes, aber doch ein reales Interesse an Meinungsumfragen besteht. Damit scheinen die Schweizer Medienschaffenden in etwa im internationalen Trend zu liegen: In einer Befragung von politischen Berichterstattern aus Frankreich und Deutschland aus dem Jahre 1988 und 1989 vermuten 74 bzw. 62 Prozent ein moderates Interesse des Publikums (Donsbach / Antoine 1990: 172). ${ }^{30}$ Darüber hinaus sind zwei Zusammenhänge festzuhalten. Zum Ersten zeigt sich, dass bei dieser allgemeinen Einschätzung des Publikumsinteresses eine Kongruenz

28 24heures, 10. November 1997.

29 Dieser hohe Validitätsanspruch wurde in 8 Prozent der 672 Artikel festgestellt. Die Kodierung lehnte sich an die von Weischenberg entwickelte Typologie von Berichterstattungsmustern an und wurde wiederum anhand der Media Polls einem Intercodierer-Reliablitätstest unterzogen. Die Identifikation erfolgte argumentativ, aufgrund einer Stringvariable. Die Zuweisung war in 92,6 \% der Fälle übereinstimmend.

30 Ein direkter Vergleich lässt sich nicht vornehmen, da die Resultate dieser Befragung in unterschiedlichen Antwortkategorien zusammengefasst sind („großes Interesse“, „moderates Interesse“ und „kleines Interesse“). In Deutschland entfallen 63\%, in Frankreich 74\% auf die mittlere Kategorie ( $\mathrm{n}=118$ bzw. 94). 
Tabelle 3: Interesse der Bevölkerung an Meinungsumfragen (Journalistenbefragung)

\begin{tabular}{|c|c|c|c|c|}
\hline & \multicolumn{2}{|c|}{$\begin{array}{l}\text { „Das Interesse der Bevölkerung } \\
\text { an repräsentativen Meinungs- } \\
\text { umfragen und Befragungs- } \\
\text { resultaten ist groß." }\end{array}$} & \multicolumn{2}{|c|}{$\begin{array}{l}\text { „Repräsentative Meinungsum- } \\
\text { fragen und Befragungsresultate } \\
\text { sind für Journalist/innen ein } \\
\text { hilfreiches Mittel bei der Erfül- } \\
\text { lung ihrer öffentlichen Aufgabe.“ }\end{array}$} \\
\hline & $\%$ & $\mathrm{n}$ & $\%$ & $\mathrm{n}$ \\
\hline 1 (stimme gar nicht zu) & 5 & 21 & 11 & 45 \\
\hline 2 & 14 & 54 & 17 & 69 \\
\hline 3 & 16 & 64 & 23 & 91 \\
\hline 4 & 30 & 118 & 30 & 121 \\
\hline 5 & 26 & 103 & 15 & 61 \\
\hline 6 (stimme voll $\mathrm{zu})$ & 9 & 36 & 4 & 18 \\
\hline \multirow[t]{2}{*}{ Total } & 100 & 396 & 100 & 405 \\
\hline & \multicolumn{2}{|c|}{ Mittelwert 3.9, Schiefe -0.37 . } & \multicolumn{2}{|c|}{ Mittelwert 3.3, Schiefe -0.10} \\
\hline
\end{tabular}

Zur Zusammensetzung des Samples (n) vgl. Fußnote 7.

Abweichungen vom Total von 100\% ergeben sich aufgrund von Rundungen.

Quelle: SPP Projekt Journalistenbefragung (Blum / Bonfadelli), eigene Berechnungen.

mit der realen Berichterstattung besteht: Befragte aus jenen Medien, welche überdurchschnittlich viele Befragungsberichte publizieren, vermuten auch bei der Leserschaft ein größeres Interesse. ${ }^{31}$ Zum Zweiten ist ein eindeutiger Zusammenhang zwischen dieser Publikumsorientierung und der generellen Einschätzung des Befragungsinstruments nachzuweisen: Wer bei der Bevölkerung ein großes Interesse vermutet, teilt eher die Ansicht, repräsentative Meinungsumfragen und Befragungsresultate würden für die Journalisten ein hilfreiches Mittel zur Erfüllung ihrer öffentlichen Aufgabe darstellen. ${ }^{32}$

31 Westschweizer Medienschaffende gehen von einem größeren Interesse der Bevölkerung aus als ihre Deutschschweizer Kollegen und Kolleginnen (Cramer's V = 0.21, p $<0.01$ Monte-CarloSignifikanz). Auch der Medientypus spielt eine Rolle bei der Einschätzung des Publikumsinteresses (Cramer's V = 0.17, $\mathrm{p}<0.01$ Monte-Carlo-Signifikanz). Das heißt, Befragte aus Re-daktionen von Sonn- und Wochenzeitungen sowie aus Agenturen gehen überdurchschnittlich häufig von einem sehr großen Interesse (Kategorie 6) der Bevölkerung an Meinungsumfragen aus. Gemäß unserer Medieninhaltsanalyse wird in der französischsprachigen Schweiz in der Tat mehr über Meinungsumfragen berichtet (vgl. auch Hardmeier 1999: 259). Auch Sonn- und Wochenzeitungen publizieren überdurchschnittlich häufig Meinungsumfragen. Gewichtet nach Anzahl erhobener Zeitungen sind es doppelt so viele wie bei Tageszeitungen: Sonn- und Wochenzeitungen publizierten während der sechsmonatigen Erhebungsperiode 59 Umfrageartikel, Tageszeitungen hingegen bloß deren 27.

32 Spearman's rho $=0.34(\mathrm{p}<0.01)$. 


\section{Präzision für die Leserschaft: Weshalb das Publikum Anspruch auf Präzision hat}

Wenn Journalistinnen und Journalisten Resultate von Meinungsumfragen publizieren, gehen viele davon aus, dass sie damit einen Beitrag zur öffentlichen Diskussion leisten. Nicht wenige sind sogar der Ansicht, dass dieser Beitrag eine direkte Wirkung hat. ${ }^{33} \mathrm{Ob}$ in Deutschland, Frankreich oder der Schweiz, deutliche Mehrheiten der befragten Medienschaffenden sind jeweils der Ansicht, publizierte Meinungsumfragen könnten einen Einfluss auf das Teilnahme- und Stimmverhalten der Bürgerinnen und Bürger haben. In der Schweiz stimmen 78 Prozent der Medienschaffenden in schreibender Funktion einer entsprechenden Aussage zu (Tabelle 4, Kategorien 4-6).${ }^{34}$ Bei jenen, welche in Befragungen ein geeignetes Mittel zur Aufgabenerfüllung sehen, wird diese Wirkungsvermutung eher noch stärker vertreten (81 Prozent). ${ }^{35}$

Unter diesen Bedingungen aber gehören Publikumsorientierung und Präzisionsanforderungen als Kriterien für die journalistische Berichterstattung zusammen: Wenn von einem Publikumsinteresse an Befragungen ausgegangen wird und Befragungsberichte als Mittel einer Aufgabenerfüllung für die Öffentlichkeit verstanden werden, dann darf an diese Befragungsberichte, die mehr als reinen Unterhaltungswert haben, auch ein entsprechender Qualitätsmaßstab gelegt werden. Wenn zudem Befragungen selbst zu einem Ereignis mit vermuteter Wirkung werden, dann sollte die Beschreibung dieses Ereignisses präzise und korrekt sein. Häufig aber verweisen die Journalisten bereits bei den Publikationsstandards auf das Publikum und begründen damit, weshalb sie die Standards nicht anwenden (de Boer 1997: 5). Die befragungstechnischen Angaben, so das Argument, würden die Verständlichkeit der Nachricht zu stark beeinträchtigen: „Solche Dinge [technische Informationen] veröffentlicht man nicht. Da wären viele Leser überfordert. [...] Es wäre viel zu schwerfällig, da würde jeder Leser aussteigen“ (Journalist F., zitiert in Flückiger et al. 1999: 89). Dieser Verweis auf die Verständlichkeit ist durchaus legitim. Denn Verständlichkeit kann als zentrales Kriterium der journalistischen Informationsqualität verstanden werden (Hagen 1995: 135). Zudem haben empirische Studien tatsächlich gezeigt, dass Nutzerinnen und Nutzer von Medien sich zum Beispiel unter dem Terminus Stichprobenfehler wenig vorstellen können (Lavrakas et al. 1991: 162 f.). Daraus jedoch die Schlussfolgerung zu ziehen, auf die Vermittlung befragungstechnischer Angaben gleich vollends zu verzichten, ist sowohl aus pragmatischen wie auch aus grundsätzlichen Überlegungen unangebracht:

33 Empirisch ist dieser Wirkungseffekt schwieriger nachzuweisen. Aufgrund der Theorie der selektiven Informationsverarbeitung wird zudem weniger von einem großen, eindeutigen NettoEffekt, sondern vielmehr von vielen verschiedenen Effekten unter diversen Bedingungen ausgegangen, vgl. Traugott (1992).

34 In der Erhebung von Donsbach und Antoine bejahten $62 \%$ der deutschen und $67 \%$ der französischen Journalisten die Frage „Do you believe that the publication of poll data in the mass media in the period before elections can have an influence on the election outcome or the voting decisions of the population?" Vgl. Donsbach / Antoine (1990: 170). In der Schweiz liegt der Anteil auch über $50 \%$, wenn lediglich die eindeutig Unterstützenden (Kategorie 5,6) einbezogen werden.

35 Dichotomisiert (Kategorien $1-3$ und $4-6$ ) ist dieser Zusammenhang noch knapp signifikant. Bei den unveränderten Daten (Kategorien 1-6) ist der Zusammenhang (Spearman's rho = 0.15) zwar nicht sehr ausgeprägt, aber signifikant $(\mathrm{p}<0.05)$. 
Tabelle 4: Wirkung von veröffentlichten Umfragedaten kurz vor Abstimmungen und Wablen (Journalistenerbebung)

\begin{tabular}{lcc}
\hline & $\begin{array}{l}\text { „Die Veröffentlichung von Umfragedaten kurz vor Abstimmun- } \\
\text { gen oder Wahlen kann die Stimmbeteiligung oder das Stimmver- } \\
\text { halten beeinflussen. }\end{array}$ & $\mathrm{n}$ \\
\hline 1 (stimme gar nicht zu) & $\%$ & 17 \\
2 & 4 & 35 \\
3 & 10 & 39 \\
4 & 24 & 94 \\
5 & 35 & 749 \\
6 (stimme voll zu) & 19 & 398 \\
Total & 100 & 74 \\
Mittelwert 4.3, Schiefe -0.773 & & \\
\hline
\end{tabular}

Abweichungen vom Total von 100\% ergeben sich aufgrund von Rundungen.

Quelle: SPP Projekt Journalistenbefragung (Blum / Bonfadelli); eigene Berechnungen.

Aus einer pragmatischen Perspektive lässt sich darauf verweisen, dass Erhebungen zur Lesbarkeit und Verständlichkeit ${ }^{36}$ von Befragungsberichten gezeigt haben, dass die Einfügung methodologischer Informationen die Texte kaum weniger lesbar macht. Nicht diese Informationen scheinen die Verständlichkeit eines Textes zu bestimmen, sondern der Schreibstil des Journalisten und seine allgemeine Einschätzung des Publikums (Duse et al. 2000: 44; de Boer 1997: 14). Zudem lässt sich das Problem der Verständlichkeit oder „Schwerfälligkeit“ - wie es Journalist F. nennt - auch mit separaten Informationskästen oder Fußnoten lösen. Dieses gestalterische Hilfsmittel hat den Vorteil, dass auf kleinem Raum und sozusagen im Telegrammstil die zentralen Angaben zur Stichprobe gemacht werden können, ohne den Textfluss zu stören.

Indessen sprechen auch grundsätzliche Überlegungen gegen eine Auslassung, und in Anlehnung an die Maximen, die in Lebensmittelverordnungen oder Landwirtschaftsgesetzen angewendet werden, lässt sich eine Deklarationspflicht für die spezifische Produktionsmethode „Befragung“ begründen: So wie nämlich in Lebensmittelverordnungen verlangt wird, dass Produkte, welche außer Sichtweite der Konsumentinnen und Konsumenten produziert oder verpackt werden, detaillierte Angaben zu Sachbezeichnung, Datierung, Zustand und Zusatzstoffen, Produzent ebenso enthalten müssen wie

36 Zur Differenzierung der Begriffe Textverständnis und Textverständlichkeit einerseits, Verständlichkeit und Lesbarkeit andererseits vgl. insbesondere auch Boss (1999). Es gilt also darauf hinzuweisen, dass in der Studie von Lavrakas et al. 1991 Verständnis - und nicht Verständlichkeit - erfasst wurde. 
eine allgemeine Gebrauchsanleitung (Lebensmittelverordnung 1999: 10), kann man sich auch eine Deklarationspflicht für Befragungen vorstellen. Wie bei den Deklarationen auf Lebensmittelverpackungen könnte zudem auch bei den Konsumierenden von Befragungsberichten von unterschiedlichen Informationsbedürfnissen ausgegangen werden. Das heißt, so wie der medienwissenschaftliche Nutzen- und Belohnungsansatz stipuliert, dass die Mediennutzer jene Informationen und Angebote auswählen, die ihren Interessen und Bedürfnissen entsprechen, wird auch die Deklaration einer Fruchtsaftflasche von „Coop“ oder bei einer Befragung gehandhabt. Auch wenn der Vermerk „2.7 mg Niacin“ oder „Stichprobenfehler \pm 3.4 Prozentpunkte“ lange nicht für alle verständlich ist - für jene, die es wissen müssen oder wollen, wird die Angabe gemacht. Sinngemäß hält ein Journalist auf die Frage nach den Publikationsstandards fest: „Ich denke, das interessiert viele Leser nicht und manche verstehen es auch nicht, aber für mich gehört es einfach dazu, für die Personen, die es interessiert. Das ist für mich Standard“" (Journalist S., zitiert in Flückiger et al. 1999: 99).

Damit aber nicht genug: Zumindest als Zielsetzung oder Ideal sei daran erinnert, dass Medien nicht nur um die Verständlichkeit, sondern auch um das Verständnis eines Berichts bemüht sein sollten. Das bedeutet, die Publikationsstandards nicht nur zu transportieren, sondern gleichzeitig auch zu erklären. Denn mit technischen Angaben einerseits, Erklärungen und Erläuterungen andererseits können beim Publikum jene Prozesse der Informationsverarbeitung ausgelöst werden, welche aus der Sozialpsychologie bekannt sind (Petty / Cacioppo 1986): Die eine Gruppe wird den Weg der systematischen Verarbeitung wählen und die präsentierten Daten und Erklärungen vertieft betrachten; die andere Gruppe wird den peripheren Weg wählen und sich an einigen Stichworten und allgemeinen Qualitätskriterien orientieren. Beide Wege können zu einer erhöhten Glaubwürdigkeit der Berichte führen. Nur der Weg über die Erklärung seitens der Medienschaffenden und die vertiefte Auseinandersetzung beim Publikum führt indessen zu dem, was zumindest langfristig als Ziel formuliert werden kann: Das sind zum einen die Lerneffekte, die sich bei den Journalistinnen und Journalisten einstellen, wenn sie mit Lesebeispielen oder Erklärungen angehalten sind, die meist in den technischen Appendix verbannten Angaben zur Qualität einer Stichprobe auf einzelne Aspekte und Fragestellungen anzuwenden. Das ist zum anderen die Situation beim Publikum, in der Befragungsberichte nicht mehr nur ein „credence good“ sind, deren Qualität allein durch die Verbreitung und Nutzung nicht beurteilt werden kann, sondern auch ein richtiges Qualitätsprodukt werden.

Einige der von uns befragten Journalistinnen und Journalisten scheinen für diese Problematik überdurchschnittlich sensibilisiert. Wer seine Rolle als eher marktorientiert und parteilich definiert, schätzt das Wissen über Demoskopie im aktuellen Berufsalltag und für die Zukunft anders ein als neutrale Berichterstatter und Berichterstatterinnen. Aufgrund der in der Journalistenbefragung erfassten Aussagen zu Berufsauffassungen und Zielvorstellungen ließen sich nämlich mittels Faktoranalyse drei Rollenverständnisse extrahieren. ${ }^{37}$ Faktor 1 steht für die „Marktorientierung “38, Faktor 2 repräsentiert

37 Die drei extrahierten Faktoren erfassen 53 \% der Varianz: Faktor $124 \%$, Faktor 2 19\% und Faktor $310 \%$. Alle 12 befragten Rollenbilder laden mit einem Wert von größer als 0.5 auf einem der drei extrahierten Faktoren.

38 Dieser Faktor umfasst die Rollenbilder Vermarkterin, Zielgruppenverkäufer, Informationsunternehmerin, Dienstleister und Animatorin. 
die „Parteilichkeit“"39 und Faktor 3 lässt sich mit „Neutralität“ umschreiben. ${ }^{40}$ Nun zeigt sich, dass zwei dieser Rollenverständnisse die Einschätzungen der Journalistinnen und Journalisten beeinflussen. Sowohl bei der Frage zur Wichtigkeit von demoskopischem Wissen im Berufsalltag als auch bei jener zur Bedeutung der sozialwissenschaftlichen Methoden in der Zukunft manifestiert sich dasselbe Muster: Je parteilicher oder je marktorientierter sich ein Journalist gibt, desto wichtiger sind Kenntnisse über Meinungsumfragen für seinen Berufsalltag ${ }^{41}$ und desto bedeutender schätzt er diese Kenntnisse für die Zukunft ein. ${ }^{42}$

Dieser Befund ist theoretisch nicht zwingend, zumal Meinungsumfragen ja auch ein wichtiges Informationsinstrument eines sachbezogenen, unparteilichen und analytischen Journalismus sein könnten, er deckt sich aber weitgehend mit dem bisher in dieser Studie gewonnenen Bild: Meinungsumfragen scheinen weniger das Instrument der Präzisions- oder Informationsjournalisten zu sein, sondern eher jenes der Unterhalterinnen und Anwälte. Das wiederum steht durchaus mit den in der Kommunikatorforschung als idealtypisch bezeichneten Rollenbildern in Einklang, und entspricht auch dem, was von Befürwortern der Demoskopie normativ postuliert wird - sofern der Umgang mit den Befragungsdaten korrekt ist:

Für Medienschaffende, die in ihren Texten Partei nehmen, können Meinungsumfragen ein wichtiges argumentatives Instrument sein. Im Sinne eines kritischen oder investigativen Journalismus ${ }^{43}$ können mit Rekurs auf demoskopisch erhobene (Mehrheits-)Meinungen Missstände oder Non-issues aufgezeigt und Meinungsumfragen als Basis für Kritik und Kommentar herangezogen werden. Damit kann unter Umständen gezeigt werden, dass die in der Öffentlichkeit besonders hörbaren Stimmen von Repräsentantinnen und Interessenvertretern nicht zwingend für jedermann sprechen (Verba 1996). ${ }^{44}$ Die Demoskopie stellt in diesem Fall eine wichtige politische Korrekturleistung dar - vorausgesetzt, das journalistische System geht professionell mit den erhobenen Daten um.

Journalistinnen und Journalisten, die mit Meinungsumfragen den Puls der Leserschaft fühlen, dienen gleichsam dem Publikum. ${ }^{45}$ In der massenmedialen Gesellschaft findet

39 Der Faktor beinhaltet die Rollenbilder Kritikerin, Kommentator, Anwältin, Vermittler und Ratgeberin.

40 Der Faktor hat die Rollenbilder von neutralen Berichterstattern und Analytikerinnen zum Inhalt.

41 Parteilichkeit: Spearman's rho, rs $=0.25, \mathrm{p}<0.01$ (Monte-Carlo-Signifikanz); Marktorientierung: $r s=0.28, \mathrm{p}<0.01$ (Monte-Carlo-Signifikanz).

42 Parteilichkeit: $r s=0.19, \mathrm{p}<0.01$ (Monte-Carlo-Signifikanz); Marktorientierung: $r s=0.22$, $\mathrm{p}<0.01$ (Monte-Carlo-Signifikanz).

43 Auch inhaltsanalytisch kann diese Art der Verwendung aufgezeigt werden. $6 \%$ der im zweiten Halbjahr 1997 erfassten Texte wurden dem investigativen Journalismus zugeordnet. Es ist allerdings bekannt, dass die berufliche Selbstdefinition von Journalisten und Journalistinnen aus verschiedenen Segmenten zusammengesetzt ist und ein pluralistisches Rollenselbstverständnis vorherrscht (vgl. Weischenberg 1995: 441). Indem das dominante Rollenbild erhoben wurde, wurde diesem Umstand annäherungsweise Rechnung getragen. In den qualitativen Interviews wird diese Verwendung einer Meinungsumfrage ebenfalls erwähnt; ein Journalist meint dazu: „Sie kann natürlich auch als Argument für den Artikel verwendet werden“. Journalist C., zitiert in Flückiger et al. (1999: 79).

44 „In general, polls can show that the noisy and overt ,representatives' of the public or of particular parts of it do not necessarily speak for everyone" (Verba 1996).

45 Dazu ein welscher Journalist: „Et puis les gens aiment bien cela [die Meinungsumfragen], ils 
nämlich Meinungsbildung der Bürgerinnen und Bürger nicht nur im persönlichen Kontakt, sondern auch über die Beobachtung der kollektiven Meinung statt. Insofern können Berichte über Meinungsumfragen ein durchaus zweckmäßiges Instrument der elektoralen Selbstbeobachtung sein (Meyer 1989: 204) 46 $^{4}$ vorausgesetzt, der Artikel hält, was er verspricht.

\section{Präzision, Publikum - und Schlagzeilen: Anregungen für eine bessere Berichterstattung}

Die Debatte über die Qualität medialer Befragungsberichte ist in einer komplexen Struktur von Angebot und Nachfrage anzusiedeln. Neben den Produzentinnen und Produzenten der Daten und den Medien als Nachfrager stehen auch die Publika - sei es als Käufer der Medien oder als potenzielle Befragte. Nicht ganz zu Unrecht wird daher häufig auch auf die unterschiedlichen motivationalen und organisationellen Imperative hingewiesen, die in diesem Akteursdreieck von Demoskopie, Journalismus und Publikum aufeinander prallen (Ladd / Benson 1992; Spinner 1998). Im Hinblick auf eine Verbesserung der aktuellen Medienberichterstattung ist dieser Hinweis indessen wenig ergiebig, denn er lädt vor allem dazu ein, vor lauter so genannten Sachzwängen fatalistisch das Handtuch zu werfen ${ }^{47}$ oder gleich zur radikalen Lösung zu greifen und nach einem Verbot zu rufen. ${ }^{48}$ Konstruktiver scheint deshalb, einmal die umgekehrte Perspektive einzunehmen und die Frage nach möglichen Berührungspunkten und gemeinsam geteilten Prämissen in Journalismus und Demoskopie zu stellen, die als Basis für eine Optimierung der medialen Berichterstattung dienen könnten. Dazu werden nachfolgend einige Anregungen formuliert. Dabei ist der Blick auf diejenigen Akteure gerichtet, die hier im Vordergrund stehen - die Medien. Komplementär dazu lassen sich Anstöße für die demoskopische Branche ablesen, die hier indessen nicht weiter ausformuliert werden.

\subsection{Die Informationsqualität der Nachrichten}

Die in Abschnitt 2.1 präsentierten Daten legen einen Bedarf bei der Aus- und Weiterbildung für die Journalistinnen und Journalisten im Hinblick auf Interpretation und Aufbereitung von Befragungsdaten nahe. Nimmt man diese Ausbildung in Angriff, sollte in einem ersten Schritt darauf hingewiesen werden, dass Handhabung und Verständnis der Publikationsrichtlinien aus der Befragungsbranche weder methodischen Purismus noch Kabbalistik bedeuten, sondern die Informationsqualität von Nachrichten tan-

aiment savoir qu'on s’intéresse à ce qu'ils pensent.“ Journalist Z., zitiert in Dufey et al. (1999: A-29).

46 „If precision journalism, in the form of pre-election and exit polls, helps the electorate to communicate with itself $(. .$.$) , then there is hope for the brave new world of direct democracy that$ mass communication technology is trying to bring us."

47 Implizit wird diese Position von jenen Autorinnen und Autoren eingenommen, welche jeden Bericht über eine Meinungsumfrage mit dem Phänomen des Horse-Race-Journalismus gleichsetzen und eine qualitativ hoch stehende Berichterstattung mit Bezugnahme auf Befragungsdaten per se in Abrede stellen (z. B. Van Praag / Van der Eijk 1998).

48 Diese Position ist vor allem unter Politikerinnen und Politikern recht prominent vertreten. Eine Untersuchung unter 78 Ländern hat jüngst ergeben, dass in $38 \%$ (30) dieser Länder gesetzliche Restriktionen hinsichtlich der Publikation von Befragungsdaten existieren (FI 1997). 
gieren und die in der Qualitätsforschung des Journalismus bekannten Kriterien der Richtigkeit, Genauigkeit, Ausgewogenheit sowie Relevanz berühren:

So kann bei den eingangs erwähnten Beispielen zu Schlagzeilen mit fraglichen Mehrheitsverhältnissen aufgezeigt werden, dass die so genannten Sonntagsfragen zu jenen seltenen Daten der Sozialforschung zählen, deren Richtigkeit ${ }^{49}$ ex post überprüft werden kann. Spätestens am Wahlabend wurde vermutlich zumindest der Autor des Artikels in der „Tribune de Genève“ an die Unschärfe ${ }^{50}$,seiner ${ }^{6}$ Erhebung erinnert: Zwar hatte er die grundsätzliche Trendwende im Kanton korrekt , prognostiziert', der faktische Stimmenanteil der Linken (Socialistes, Verts und Alliance de gauche) lag indessen gerade unter der absoluten Mehrheit der gültigen Stimmen (47,2 \%). ${ }^{51}$

Richtigkeit ist indessen nur in seltenen Fällen überprüfbar, weshalb Genauigkeit und Ausgewogenheit als Kriterien für die Informationsqualität von Bedeutung sind. Dabei verlangt die Norm der Genauigkeit eines Berichts über Befragungen vor allem, dass der Unschärfebereich einer Stichprobe bei der Interpretation der Daten berücksichtigt wird und auf Angaben auf die Kommastelle genau verzichtet wird, weil diese eine Punktgenauigkeit suggerieren, die nicht einlösbar ist. Eine ausgewogene Berichterstattung wiederum impliziert eine angemessene Berücksichtigung unterschiedlicher Positionen oder Meinungen. Hier ist die Demoskopie im Gegensatz zum Journalismus in der glücklichen Lage, dass für diese Norm einer adäquaten Realitätsbeschreibung auch ,harte' Indikatoren zur Verfügung stehen. Anhand von Signifikanztests und Zusammenhangsmassen lassen sich nämlich Unterschiede zwischen Gruppen im Hinblick auf Stärke und Ausprägung überprüfen. So kann im aufgeführten Beispiel aus der „Berner Zeitung“ davon ausgegangen werden, dass der Bericht nicht ausgewogen ist. Vielmehr wird hier eine Position - nämlich die des Gegensatzes zwischen Deutschschweiz und Romandie - vertreten, welche nicht signifikant ist, dafür viel eher die Nachrichtenwerte Negativismus oder Konflikt sowie Kontinuität (des so genannten Röstigrabens zwischen Deutschschweiz und Romandie) befriedigt.

Bei den zwei Beispielen zum problematischen Umgang mit relativen Mehrheiten geht es vor allem auch um die Relevanz ${ }^{52}$ der medial vermittelten Informationen, die in den öffentlichen und individuellen Meinungsbildungsprozess einfließen können: So stellt sich beim Beispiel der Luzerner Verkehrsbefragung die Frage, ob hier der Journalist

49 In vielen normativen und empirischen Systematiken zur Objektivität von Berichterstattung wird Richtigkeit als Kriterium „deskriptiver Qualität“ (Schatz / Schulz 1992: 705 ff.) verwendet. $\mathrm{Da}$ in modernen Informationsgesellschaften die Wahrhaftigkeit von Aussagen kaum mehr direkt zu eruieren ist, müssen Bewertungen zur Qualität von Aussagen unter Unsicherheit vorgenommen werden. Richtigkeit ist ein Maßstab dafür und er kann in die Dimensionen Wahrheit und dem daraus abgeleiteten Begriff der Genauigkeit („möglichst dicht an der Wahrheit“) unterteilt werden.

50 Auf die anderen Schwierigkeiten bei Befragungen vor Wahlen in einem Proporzsystem sei hier gar nicht eingegangen.

51 Erst auf der Ebene der Sitze konnte eine knappe Mehrheit von 51 von 100 Sitzen verbucht werden.

52 Relevanz gilt ebenfalls als wichtiges Kriterium der Informationsqualität (Hagen 1995: 70). Deshalb wird in der Kommunikationswissenschaft analysiert, weshalb einem Faktum mehr Aufmerksamkeit geschenkt wird als einem anderem. Und wenn dabei beispielsweise Schatz und Schulz (1992) auch die „Zahl der Betroffenen“ als zentrales Kriterium für Relevanz aufführen, dann wird die Parallele zu unseren Beispielen, wo es um absolute und relative Mehrheiten geht, offensichtlich. Insofern stellt sich immer die Frage, ob ein Fakt oder ein Prozentsatz relevant genug ist, um ihm die Aufmerksamkeit zu schenken und eine Nachricht darauf aufzubauen. 
nicht einem in den Medien beliebten Diskurs über die vermeintliche Unzufriedenheit der Bürgerschaft aufsitzt und ein Erzählschema aktiviert, das einen Konflikt zwischen Regierenden und Regierten konstruiert, der in der Realität bzw. in den Befragungsdaten in diesem Ausmaß gar nicht vorhanden ist. Gleichermaßen lässt sich gegenüber der journalistischen Aufbereitung der Arbeitszeit-Befragung einwenden, dass im Hinblick auf die Chancen des 1998 eingereichten Volksbegehrens für eine 36-Stunden-Woche ein realitätsfremdes Bild gezeichnet wird. Auch die Problematik von divergierenden Grund- und Erhebungsgesamtheiten kann aus der Perspektive des journalistischen Gütekriteriums Relevanz betrachtet werden, insofern als sich hier die Frage der tatsächlich Betroffenen und involvierten Akteure stellt. So kann die mangelnde journalistische Präzision Ursache von prognostischen Fehleinschätzungen werden, wenn bei einer bevorstehenden Wahl oder Abstimmung das Interesse ohnehin „nur“ bei den potenziellen Urnengängerinnen und Urnengängern liegt - und in der Stichprobe auch entsprechend diese Zielpopulation erfasst wurde. Oder es kann in einer multikulturellen Gesellschaft zu leichten Irritationen führen, wenn nur ein Teil der Staatsangehörigen befragt wurde, aber von ,Schweizerinnen und Schweizern' die Rede ist. Schließlich wird es normativ störend, wenn bei einer Stichprobe, in welcher rund 20 Prozent Ausländerinnen und Ausländer explizit ausgeklammert werden, von ,Bevölkerung' die Rede ist. In diesem Fall bleibt nämlich gerade ein demokratischer Anspruch der Demoskopie uneingelöst, der häufig formuliert wird: jenen eine Stimme zu geben, die im institutionellen Entscheidprozess keine Stimme haben.

\subsection{Handwerksregeln für die Präsentation und Aufbereitung}

Sowohl Journalismus wie auch Demoskopie wollen Wissen produzieren und vermitteln - sei es in Form von Nachrichten oder in Form von Befragungsdaten. Und wenn Nachrichten über Befragungen produziert werden, überschneiden sich die Kriterien für diese Wissensvermittlung. Für den Journalismus bedeutet das: Wenn man sich entschieden hat, aus Befragungsberichten und Resultaten von Meinungsumfragen einen medialen Bericht zu verfassen, dann gilt grundsätzlich, dass die Befragung ein Ereignis darstellt wie jedes andere auch. Ebenso ist das Produkt eine Nachricht wie jede andere journalistische Mitteilung auch. Deshalb können die berühmten, in jedem Praxishandbuch des Journalismus zitierten 7 W-Fragen für eine Nachricht auch auf jede Befragung angewendet werden. Und der in Tabelle 5 dargestellte Vergleich zeigt, dass die journalistischen Handwerksregeln für die Präsentation und Aufbereitung von Nachrichten große Parallelen mit jenen in der Demoskopie aufweisen (Hardmeier 2000). Grundsätzlich werden nämlich die gleichen Fragen gestellt. Sowohl in Demoskopie wie auch im Journalismus existieren Regeln für die Selektion von Daten bzw. Ereignissen, werden Handlungsanleitungen für die Interpretation von Befunden bzw. Nachrichten formuliert und gibt es Richtlinien zur Transparentmachung der Erhebung bzw. Quelle.

Bei der Präsentation von Befragungsergebnissen können separate Kästchen (Infobox) oder Fußnoten helfen, die wichtigsten Parameter einer Stichprobe auf begrenztem Raum zu präsentieren (vgl. Tabelle 1). Ein mehrseitiger methodischer Bericht kann so verdichtet werden, dass er mit ein paar wenigen Zeilen in einem Informationskasten oder einer Fußnote eingefügt werden kann. Das häufig aufgeführte Argument der Platzrestriktionen ist daher eigentlich nur für Kurzmeldungen zulässig, welche meistens in Form einer Sekundärberichterstattung in ein paar Zeilen über eine Befragung berichten, von der man über PR-Kanäle (Pressekonferenzen, Mitteilungen etc.) oder über Presseagenturen in Kenntnis gesetzt wurde (Hardmeier 1999: 265). Würde man in diesen Mel- 


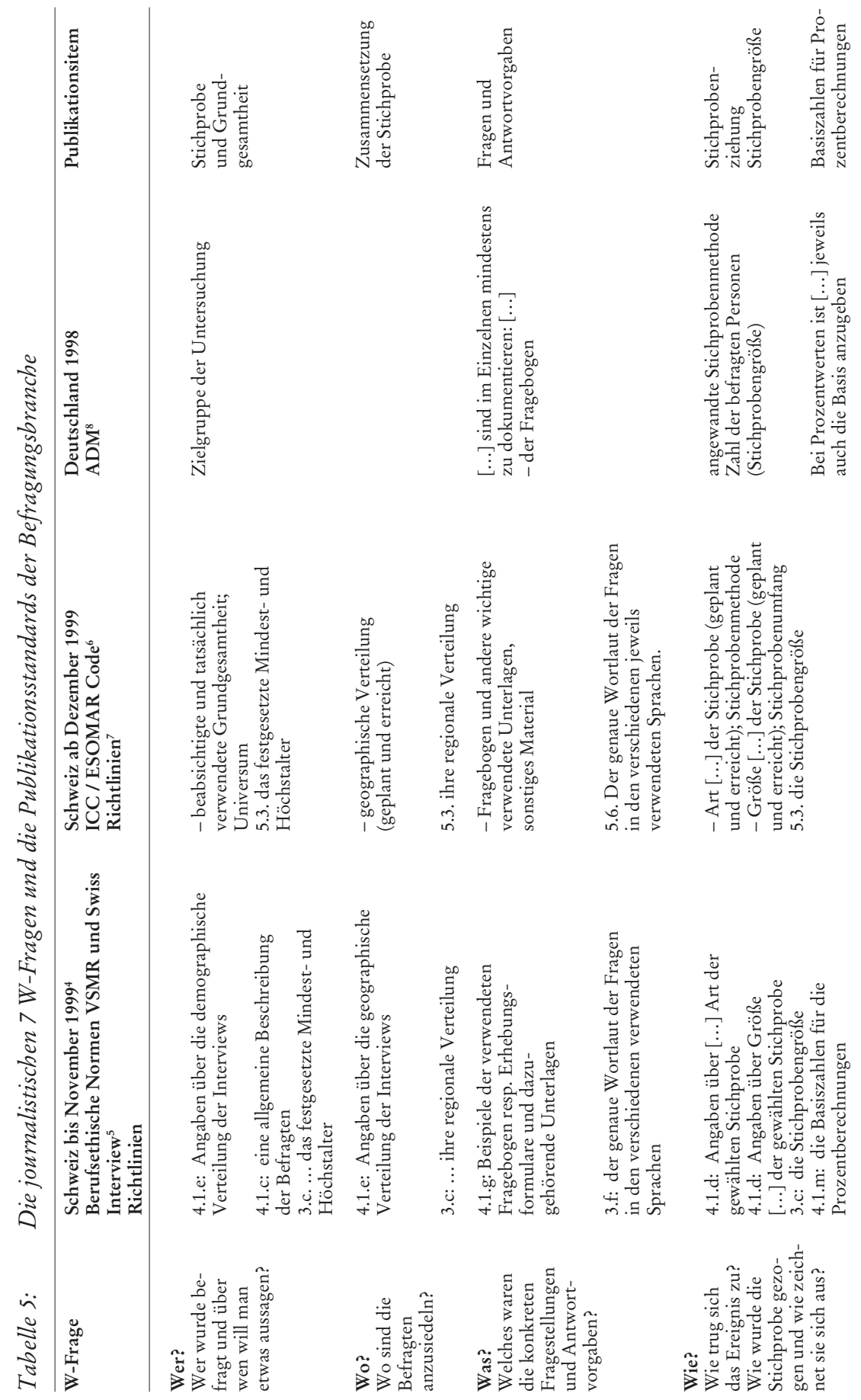




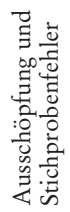
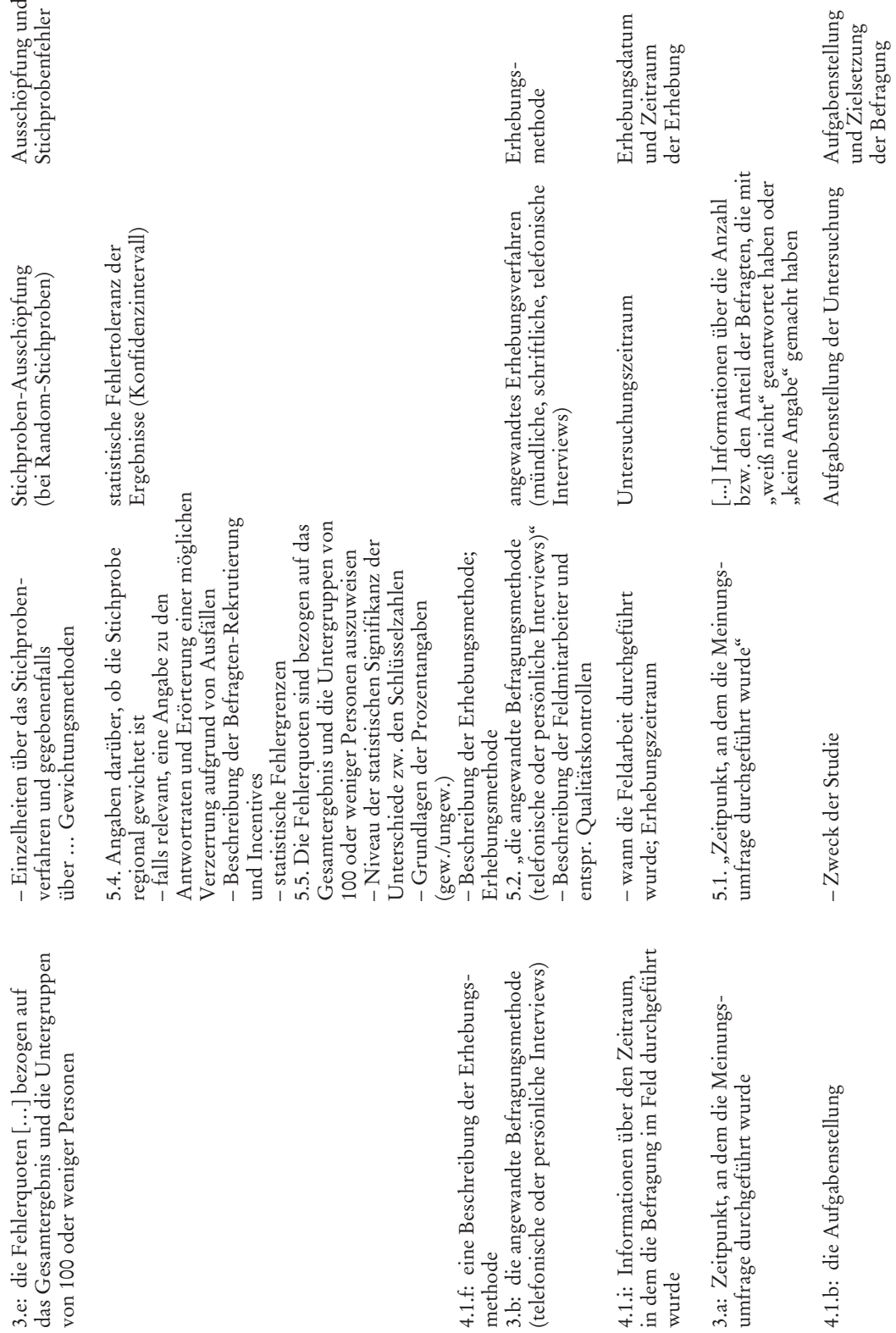

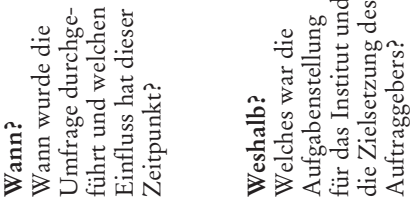

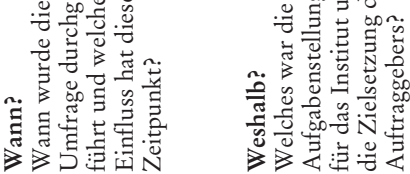




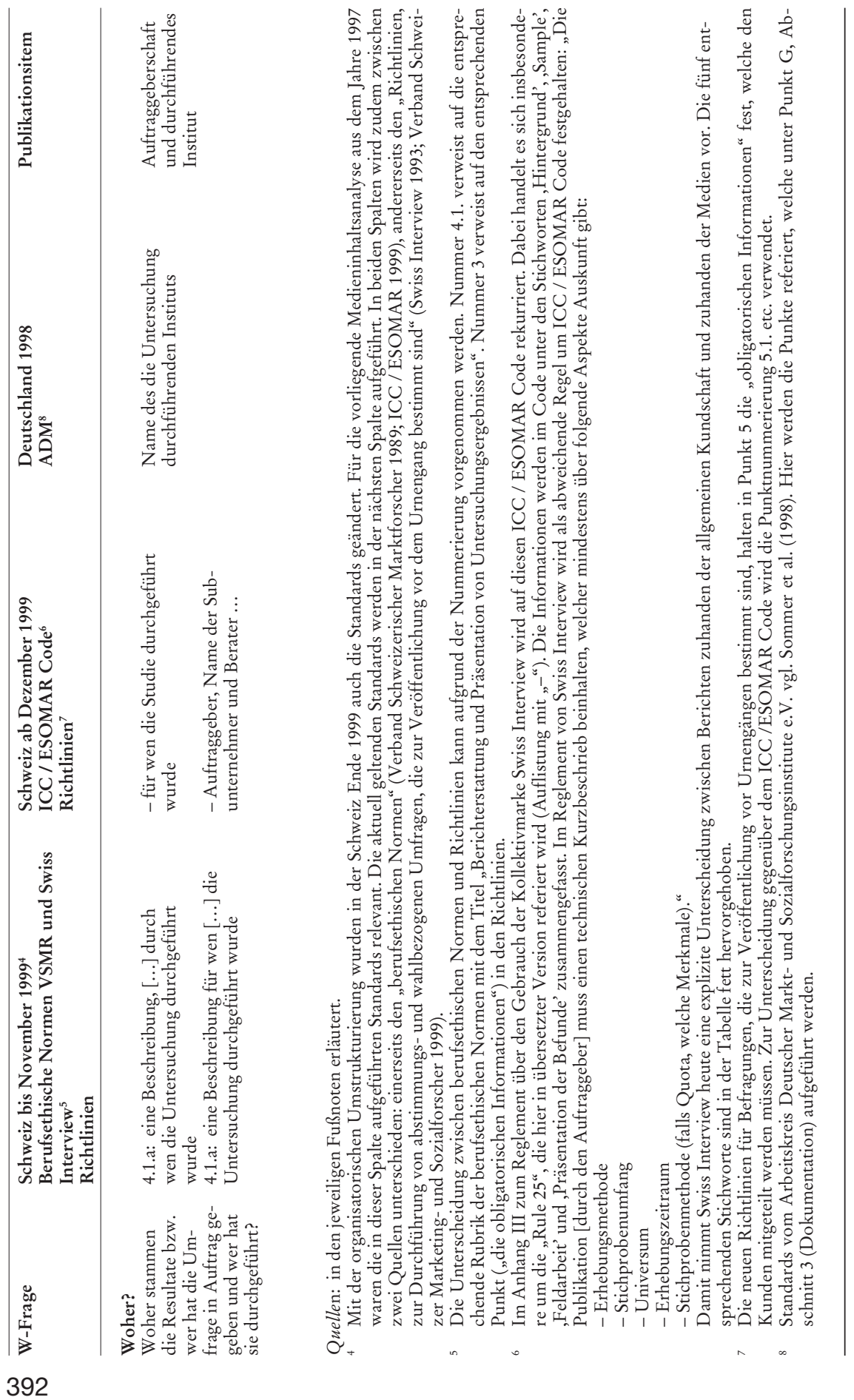


dungen die Publikationsstandards und die 7 W-Fragen vermitteln, wäre der diesbezügliche Abschnitt in der Tat länger als die Meldung selbst. Aber auch für dieses Dilemma lässt sich heute eine Lösung finden. Kurzmeldungen könnten mit einem Qualitätslabel versehen werden, und mit einer www-Adresse kann auf die ausführlichen Informationen und Dokumentationen hingewiesen werden. Im Falle eines von Associated Press (AP) verbreiteten Befragungsresultates würde der Vermerk zum Beispiel lauten: „APproofed; vgl. www.associatedpress.ch/umfrage_bilateralevertraege“. Falls das Material bzw. die www-Adressen nicht von den Vermittlungsinstanzen selbst angeboten wird, sollte das publizierende Medium eine entsprechende Aufbereitung vornehmen. Die Mitteilung würde dann in etwa lauten: „von der Tageszeitung als seriös beurteilt; vgl. www.tageszeitung.ch/umfrage_bilateralevertraege“. Dort wiederum, wo die Zeit für eine solche Überprüfung nicht ausreicht oder die Qualitätsanalyse zu einem negativen Befund kommt, ist ein Verzicht auf die Publikation wohl der angemessenste Schritt.

\subsection{Selektionsregeln für Schlagzeilen}

Gegenüber dem Ansinnen, die journalistische Aus- und Weiterbildung für mehr Präzision und Publikumsorientierung bei Befragungsberichten voranzutreiben, kann der Einwand erhoben werden, diese ,hehre' Absicht widerspreche der konstruktivistischen Funktionslogik der Medien, News zu produzieren..$^{53}$ Der Einwand ist nicht unberechtigt, und exemplarische Fallstudien oder linguistische Analysen können diese Mechanismen durchaus aufzeigen (Hardmeier / Luginbühl 2000). An der Aus- und Weiterbildung scheint aber dennoch kein Weg vorbeizuführen: Während nämlich die einzelnen Medienschaffenden den ökonomischen Druck für Schlagzeilen kaum beeinflussen können, sind sie als Individuen in der Lage, etwas für präzise Schlagzeilen zu tun. Jede Befragung verfügt nämlich über einen Nachrichtenwert. Die Frage ist nur, welchen Nachrichtenfaktor man aktiviert und wie man den Nachrichtenwert entdeckt - ob über vorgefasste Meinungen oder über eine präzise Analyse der Daten: Wenn die Journalistinnen und Journalisten über Effekte von Frageformulierungen informiert sind, können sie Relevanz und Aussagekraft von Ergebnissen besser einschätzen. Wenn sie für die Unschärfe von Stichprobenziehungen sensibilisiert sind, werden sie von Prozentzahlen mit Kommastellen ablassen, dafür aber ihren Blick für absolute und relative Mehrheitsverhältnisse schärfen. Wenn sie keine Veränderung über das Vertrauensintervall hinaus entdecken, entscheiden sie sich vielleicht eher für den Nachrichtenwert „Kontinuität“als für jenen der „Überraschung“. Und wenn sie Signifikanzniveau und Zusammenhangsmasse interpretieren können, berichten sie über jene Konflikte in gesellschaftlichen Gruppen, die in der Realität auch tatsächlich existieren.

\section{Literatur}

Berger, Peter, L. / Berger, Brigitte (1983): Wir und die Gesellschaft. Eine Einführung in die Soziologie - entwickelt an der Alltagserfahrung, Stuttgart.

Boss, Peter (1999): Faktoren der Textverständlichkeit, Zürich (mimeo).

53 „Wir gehen dabei [bei der Generierung der Nachricht] nach journalistischen Kriterien, nicht nach wissenschaftlichen vor. Wir nehmen das spannendste Element aus den Resultaten heraus und fokussieren darauf. (...) In diesem Falle muss es ein überraschendes Element sein und relevant sein." Journalist C., zitiert in Dufey et al. (1999: A-20 f.). 
Brady, Henri E. / Orren, Gary R. (1992): „Polling Pitfalls: Sources of Error in Public Opinion Surveys“. In: Thomas E. Mann / Orren, Gary R. (eds.), Media Polls in American Politics, Washington, D.C., 55 - 94.

Brettschneider, Frank (1997): „The Press and the Polls in Germany, 1980 - 1994“, International Journal of Public Opinion Research, 9, 248 - 65.

de Boer, Connie (1995): Peilingen in de pers. Een studie naar het gebruik van opiniepeilingen in de Nederlandse dagbladen in de periode 1960 - 1988, Proefschrift Universiteit van Amsterdam.

de Boer, Connie (1997): Opinion Polls in Newspapers: Readability and the Presentation of Methodological Information, Paper presented at the WAPOR 50th Anniversary Conference, Edinburgh, Scotland, September $11-12,1997$.

Donsbach, Wolfgang (1991): „Objektivitätsmasse in der Publizistikwissenschaft“, Publizistik, Heft $1,18-29$.

Donsbach, Wolfgang / Antoine, Jacques (1990): „Journalists and the Polls: A Parallel Survey among Journalists in France and Germany“, Marketing and Research Today, August 1990, 167 - 174.

Dufey, Gisella et al. (1999): Media Polls. Journalistisches Framing und Ereignismanagement, Zürich (mimeo).

Duse, Eva et al. (2000): Readability. Faktoren der Textverständlichkeit. Eine Untersuchung zur Verständlichkeit von Artikeln über politische Meinungsumfragen in Schweizer Printmedien, Zürich (mimeo).

FI / ESOMAR / WAPOR (eds.) (1997): The Freedom to Publish Opinion Polls. Report on a Worldwide Study, Amsterdam.

Flückiger, Peter et al. (1999): Die Publikation von Meinungsumfragen in Schweizer Printmedien unter der Lupe. Journalistische Berichterstattung und Präsentation von Untersuchungsergebnissen in Deutschschweizer Printmedien, Zürich (mimeo).

Groves, Robert M. (1989): Survey Errors and Survey Costs. New York etc.

Hagen, Lutz M. (1995): Informationsqualität von Nachrichten. Messmethoden und ihre Anwendung auf die Dienste von Nachrichtenagenturen, Opladen.

Hardmeier, Sibylle (1999): „Political Poll Reporting in Swiss Print Media: Analysis and Suggestions for Improvement", International Journal of Public Opinion Research, Vol. 11, No. 3, 257-274.

Hardmeier, Sibylle (2000): Communicating Survey Quality Criteria: Towards a new guideline for poll reporting, Paper prepared for the WAPOR Seminar, Cadenabbia 2000, June 29 to July 1, 2000 „Intellectual and content-related aspects of quality criteria in survey research“ (in cooperation with Connie de Boer, mimeo).

Hardmeier, Sibylle / Luginbühl, Martin (2000): „Zur Informationsqualität von Zeitungsberichten über Meinungsumfragen: eine exemplarische Studie zur medialen Konstruktion von Realität“, Publizistik, Heft 2, Juni, $180-199$.

ICC / ESOMAR (1999) (ed.): ICC / ESOMAR International Code of Marketing and Social Research Practice, www.esomar.nl/codes_1.html.

Kaase, Max (1994): „Demoskopie / Umfrageforschung“. In: Nohlen, Dieter (Hrsg.), Lexikon der Politik, Bd. 2 Politikwissenschaftliche Methoden, 71 - 76.

Ladd, Everett Carll / Benson, John (1992): „The Growth of News Polls in American Politics“. In: Thomas E. Mann / Orren, Gary R. (eds.), Media Polls in American Politics, Washington, D. C., $19-31$.

Lavrakas Paul J. et al. (1991): „Public Reactions to Polling News During the 1988 Presidential Election Campaign" In: Lavrakas, Paul J. / Holley, Jack K. (eds.), Polling and Presidential Election Coverage, Newbury Park, 151 - 183.

Lebensmittelverordnung (1999): Lebensmittelverordnung LMV vom 1. März 1995, Stand am 23. November 1999, Systematische Rechtssammlung der Schweizerischen Eidgenossenschaft SR 187.02

Marr, Mirko / Wyss, Vinzenz (1999): „Schweizer Journalistinnen und Journalisten im sprachregionalen Vergleich: Merkmale und Einstellungen“, Medienwissenschaft Schweiz, Heft 2, 16 30.

Meyer, Philip (1989): „Precision Journalism and the 1988 US Elections“, International Journal of Public Opinion Research, Vol. 1, No. 3, 195 - 205. 
Meyer, Philip (1991): The New Precision Journalism, Bloomington / Indianapolis.

Miller, Mark M. / Hurd, Robert (1982): „Conformity to AAPOR Standards in Newspaper Reporting of Public Opinion Polls“, Public Opinion Quarterly, Vol. 46, 243 - 249.

Miller, Peter V. et al. (1991): „Journalism with Footnotes. Reporting the ,Technical Details‘ of Polls“. In: Lavrakas, Paul J. / Holley, Jack K. (eds.), Polling and Presidential Election Coverage, Newbury Park, $200-214$.

Mutz, Diana C. (1998): Impersonal Influence. How Perceptions of Mass Collectives Affect Political Attitudes, Cambridge UK.

Neidhardt, Friedhelm (1994): „Öffentlichkeit, öffentliche Meinung, soziale Bewegungen“. In: Neidhardt, Friedhelm (ed.), Öffentlichkeit, öffentliche Meinung, soziale Bewegungen, Sonderheft Kölner Zeitschrift für Soziologie und Sozialpsychologie, Nr. 34, Opladen, 7 - 41.

Paletz, David L. et al. (1980): „Polls in the Media: Content, Credibility, and Consequences“, Public Opinion Quarterly, No. 44, 495 - 513.

Petty, Richard E. / Cacioppo, John T. (1986), „The Elaboration Likelihood Model of Persuasion“. In: Berkowitz, Leonard (ed.), Advances in Experimental Social Psychology, Bd. 3, New York, $123-205$.

Rollberg, Jeanne Norton et al. (1990): „Down to the wire. How six newspapers reported public opinion polls during the 1988 presidential campaign", Newspaper Research Journal, Vol. 11, no. 4, $80-93$.

Schatz, Heribert / Schulz, Winfried (1992): „Qualität von Fernsehprogrammen. Kriterien und Methoden zur Beurteilung von Programmqualität im dualen Fernsehsystem“, Media Perspektiven $11,690-712$.

Salwen, Michael B. (1985): „The Reporting of Public Opinion Polls During Presidential Years, 1968 - 1984“, Journalism Quarterly, Vol. 62, 272 - 277.

Spinner, Helmut F. (1998): Die Architektur der Informationsgesellschaft: Entwurf eines wissensorientierten Gesamtkonzepts, Bodenheim.

Stierli, Markus (2000): Media Polls in der Schweizerischen Presselandschaft. Quantitative Analyse von Qualität, Diffusion und Transformation, Zürich (mimeo).

Stovall, James Glen / Solomon, Jacqueline H. (1984): „The Poll as a News Event in the 1980 Presidential Campaign“, Public Opinion Quarterly, 48, 615 - 23.

Swiss Interview (1993): „Richtlinien zur Durchführung von abstimmungs- und wahlbezogenen Umfragen, die zur Veröffentlichung vor dem Urnengang bestimmt sind“. In: Swiss Interview (Hrsg.): Edition 93, Zahlen und Fakten, Luzern.

Traugott, Michael (1992): „The Impact of Media Polls on the Public“. In: Mann, Thomas E. / Orren, Gary R. (eds.), Media Polls in American Politics, Washington, D.C., 125 - 149.

Van Praag, Philip / Van der Eijk, Cees (1998): „News Content and Effects in a Historic Campaign“, Political Communication, Vol. 15, No. 1, 165 - 185.

Verba, Sidney (1996): „The Citizen as Respondent: Sample Surveys and American Democracy“, American Political Science Review, Vol. 90, No. 1, 1-7.

Verband Schweizer Marketing- und Sozialforscher (2000): Marketing \& Sozialforschung Schweiz, Jahrbuch 2000, Berneck.

Verband Schweizer Marketing- und Sozialforscher (1999): Richtlinien zur Durchführung von abstimmungs- und wahlbezogenen Umfragen, die zur Veröffentlichung vor dem Urnengang bestimmt sind. Anhang IV zum Reglement über den Gebrauch der Kollektivmarke Swiss Interview, Zürich/Cham (mimeo).

Verband Schweizerischer Marktforscher und Swiss Interview (1989): „Berufsethische Normen des VSMF und der SWISS INTERVIEW“. In: Verband Schweizerischer Marktforscher - VSMF (Hrsg.): Marktforschung Schweiz Handbuch 90/91. Dietikon, S. 153.

Weimann, Gabriel (1990): „The Obsession to Forecast: Pre-Election Polls in the Israeli Press“, Public Opinion Quarterly, 54, 396 - 408.

Weischenberg, Siegfried (1995): Journalistik, Theorie und Praxis aktueller Medienkommunikation, Band 2: Medientechnik, Medienfunktion, Medienakteure, Opladen/Wiesbaden. 